UUITP-05/11

MAD-TH-10-04

\title{
De Sitter hunting in a classical landscape
}

\author{
Ulf H. Danielsson ${ }^{a}$, Sheikh S. Haque ${ }^{b}$, Paul Koerber ${ }^{c}$, \\ Gary Shiu ${ }^{b, d}$, Thomas Van $\operatorname{Riet}^{a}$ and Timm Wrase ${ }^{d, e}$ 1 \\ ${ }^{a}$ Institutionen för Fysik och Astronomi, \\ Box 803, SE-751 08 Uppsala, Sweden \\ ${ }^{b}$ Department of Physics, University of Wisconsin, \\ Madison, WI 53706, USA \\ ${ }^{c}$ Instituut voor Theoretische Fysica, K.U.Leuven, \\ Celestijnenlaan 200D, B-3001 Leuven, Belgium \\ ${ }^{d}$ Institute for Advanced Study, Hong Kong University of Science and Technology, \\ Hong Kong, People's Republic of China \\ e Department of Physics, Cornell University, \\ Ithaca, NY 14853, USA
}

\begin{abstract}
We elaborate on the construction of de Sitter solutions from IIA orientifolds of SU(3)structure manifolds that solve the 10-dimensional equations of motion at tree-level in the approximation of smeared sources. First we classify geometries that are orbifolds of a group manifold covering space which, upon the proper inclusion of O6 planes, can be described within the framework of $\mathcal{N}=1$ supergravity in $4 \mathrm{D}$. Then we scan systematically for de Sitter solutions, obtained as critical points of an effective 4D potential. Apart from finding many new solutions we emphasize the challenges in constructing explicit classical de Sitter vacua, which have sofar not been met. These challenges are interesting avenues for further research and include finding solutions that are perturbatively stable, satisfy charge and flux quantization, and have genuine localized (versus smeared) orientifold sources. This paper intends to be self-contained and pedagogical, and thus can serve as a guide to the necessary technical tools required for this line of research. In an appendix we explain how to study flux and charge quantization in the presence of a non-trivial $H$-field using twisted homology.
\end{abstract}

\footnotetext{
${ }^{1}$ ulf.danielsson at physics.uu.se, haque at wisc.edu, koerber at itf.fys.kuleuven.be, shiu at physics.wisc.edu, thomas.vanriet at fysast.uu.se, timm.wrase at cornell.edu
} 


\section{Contents}

1 Introduction 3

2 The 4D and 10D picture

2.1 Dimensional reduction and effective $4 \mathrm{D}$ theories . . . . . . . . . . 5

2.2 The "universal" ansatz in 10D . . . . . . . . . . . . . . . . 9

3 Classification of geometries $\quad 11$

3.1 Homogenous SU(3)-structures . . . . . . . . . . . . . . . . . . . . 11

3.2 Group manifold geometry and geometric moduli . . . . . . . . . . . . . . . . 13

3.3 All unipotent real six-dimensional Lie algebras . . . . . . . . . . . . . . . 14

4 Discrete symmetries, orbifolds and orientifolds 18

4.1 Discrete subgroups of $\mathrm{SU}(3) \ldots \ldots \ldots \ldots \ldots$. . . . . . . . . . . . . . . .

4.2 The standard $\mathbb{Z}_{2} \times \mathbb{Z}_{2}$ orientifold example . . . . . . . . . . . . . . . . . 20

4.3 The non-standard $\mathbb{Z}_{2} \times \mathbb{Z}_{2}$ orientifold example . . . . . . . . . . . . 24

4.4 General abelian and non-abelian orbifolds . . . . . . . . . . . . 26

4.4.1 The standard orientifold of $\Delta(12) \ldots \ldots \ldots \ldots$. . . . . . . . . . . . . . . . . . . .

4.4 .2 The non-standard orientifold of $\Delta(12) \ldots \ldots \ldots$

5 Classification of de Sitter solutions $\quad 28$

5.1 The standard $\mathbb{Z}_{2} \times \mathbb{Z}_{2}$ orientifold . . . . . . . . . . . . . . . . . 29

5.2 The non-standard $\mathbb{Z}_{2} \times \mathbb{Z}_{2}$ orientifold . . . . . . . . . . . . . . 31

5.3 The non-abelian orbifold $\Delta(12) \ldots \ldots \ldots \ldots \ldots \ldots \ldots$

5.4 The mass spectrum . . . . . . . . . . . . . . . . . . . . . . . . 33

6 Flux and charge quantization 34

6.1 Quantization of the $\mathrm{dS}$ solution on $\mathrm{SU}(2) \times \mathrm{SU}(2) \ldots \ldots \ldots$

7 Discussion 35

A Form conventions for reduction to 4D 38

B Half-flat manifolds 38

C 10D equations of motion for the universal ansatz 40

D Flux/charge quantization with non-trivial $H$-field 41

D.1 General discussion . . . . . . . . . . . . . . . . . . . . 41

D.2 The $H$-twisted homology of $\mathrm{SU}(2) \times \mathrm{SU}(2) \ldots \ldots \ldots \ldots$ 


\section{Introduction}

The increasing experimental support for an accelerating universe also presents a great challenge for quantum gravity. The simplest explanation for this cosmic puzzle is that we are living in a de Sitter universe, i.e. a (metastable) vacuum with a positive cosmological constant. While the zero of the vacuum energy is immaterial in the absence of gravity, and the cosmological constant can be tuned at will classically, it is not so in the context of quantum gravity. Thus, finding an explicit de Sitter vacuum which matches with (and hopefully explains) observations is arguably a holy grail for any candidate quantum theory of gravity, such as string theory.

Like the search for the Holy Grail, the search for de Sitter vacua in string theory has proven to be an elusive quest. Before taking into account the full quantum corrections to the cosmological constant, the construction of de Sitter vacua even at leading orden 2 (i.e. within classical supergravity) is complicated by many additional issues, such as moduli stabilization (and vacuum stability), flux quantization, and a proper microscopic treatment of localized sources. These issues, all in one way or the other related to the fact that string theory comes with extra dimensions of space, may well turn out to give us important leads for finding de Sitter vacua in a fundamental theory of gravity.

Particularly on the issue of moduli stabilization, the difficulty it imposes on constructing de Sitter vacua is sharpened by several no-go theorems. These theorems were presented in the language of $4 \mathrm{D}$ effective field theories: by requiring the existence of a positive-energy extremum of the potential in the dilaton and breathing mode direction [4-12], one finds necessary conditions for obtaining de Sitter vacua within classical supergravity. We refer to de Sitter backgrounds obtained within supergravity, with the possibility of localized sources and fluxes, but without introducing stringy corrections or non-perturbative effects, as classical de Sitter solutions. Attempts to construct actual classical de Sitter solutions from $4 \mathrm{D}$ effective field theory were made in [5,7, 9, 11, 13 17] (see also [18]). Moreover, not many of such 4D solutions were demonstrated to arise as full supergravity backgrounds, especially those that break supersymmetry at the Kaluza-Klein scale [5, 7, 13, 16, 17]. This motivated one to construct classical de Sitter solutions directly in 10 dimensions [10, 19] 3] The work of [19] explicitly mapped a solution obtained in 10D to a solution obtained in [8] using $4 \mathrm{D}$ techniques. This solution has a simple and elegant form in the full 10D description and will be used in this paper to investigate charge and flux quantization.

In this paper, we systematically explore the possibility of constructing classical de Sitter vacua. Armed with the insights from previous attempts, we consider Type IIA orientifolds

\footnotetext{
${ }^{2}$ One can consider de Sitter vacua that arise only after non-perturbative effects are included, as is often assumed in heterotic string theory or in the KKLT scenario 1. However, the uplift to de Sitter space requires anti-branes [1] or stringy corrections [2,3] whose explicit microscopic description may invoke similar issues encountered here.

${ }^{3}$ Here, we mention in passing that compactifications with non-geometric fluxes seem to lead to stable de Sitter vacua [14,15, though it is not clear if a supergravity approach is reliable for such constructions. Finally, the aim of the present work is to search for de Sitter vacua from compactifications of string theory. We refer the readers to 20,24 for some non-compact examples.
} 
of SU(3)-structure manifolds. In particular, we classify SU(3)-structure manifolds that are realized as orbifolds of a group (or coset) manifold as the analysis is much simplified for homogeneous spaces 4 We are particularly interested in compactifications which do not break supersymmetry at the Kaluza-Klein scale 5 The de Sitter solutions so obtained are critical points of an effective $4 \mathrm{D}$ potential; supersymmetry is broken spontaneously rather than explicitly and thus the de Sitter backgrounds are under better analytic control. However, the on-shell value for the volume modulus might be such that the KK scale is of the same order as the flux-induced masses (which roughly give the SUSY breaking scale). Then one has to worry about the consistency of the effective potential. But since we restrict to left-invariant modes on homogenous spaces, the dimensional reduction is mathematically consistent and solutions of the four-dimensional action lift to solutions of the ten-dimensional action [30].

Moduli stabilization as well as flux and charge quantization are subjects of concern in this paper, but we leave the challenge of finding de Sitter backgrounds with account of the back-reaction of localized sources for future work. As in the previous literature on the subject, we will therefore look for supergravity solutions where the O-planes are smeared over their transverse directions [6, 31-34]. One can think of the "smeared" approximation as solving the equations of motion in an average sense, even though they are not satisfied pointwise in the internal space $33-35]$.

An important obstacle for all the known dS solutions in these compactifications is that none sofar are perturbatively stable. This is why one needs to increase the number of models, as we do here, in the hope to find a perturbatively stable solution. Unfortunately all new solutions we find in this paper have at least one tachyonic mode in the left-invariant spectrum.

The paper is organized as follows. In section 2, we discuss the reduction of type IIA string theory on $\mathrm{SU}(3)$ structure manifolds, providing both a 4D and a $10 \mathrm{D}$ perspective. We emphasize the utility of the "universal" ansatz in the search for explicit solutions. In section 3, we classify homogenous geometries that are consistent with an SU(3)-structure. Our classification of group (and coset) manifolds in this section subsumes all the previously found examples in the literature and furthermore provides a unified, group theoretical understanding of these previous works. Section 4 discusses orbifold and orientifold projections and contains some explicit examples of group spaces with $\mathrm{SU}(3)$-structure. We then apply the results of the previous two sections to explore de Sitter solutions in section 5 . In addition to solving the equations of motion - which are local conditions - tadpole cancelation and quantization of charges and fluxes impose additional, global, constraints. We discuss these global constraints in section 6 using twisted homology [36]. We explicitly quantize the charge and fluxes of the solution on $\mathrm{SU}(2) \times \mathrm{SU}(2)$, presented in [8, 19] and shall see that this solution does not survive quantization. Therefore quantization can serve

\footnotetext{
${ }^{4}$ For this reason coset and group manifolds were already studied early on, for instance in the context of the Kaluza-Klein approach to unification. For reviews see [25, 26, and references therein. For other early work, e.g. in the context of heterotic string theory, see [27 29].

${ }^{5}$ More precisely, this means we consider compactifications that do not include explicit SUSY breaking orientifold sources, so that SUSY is broken by the (geometric) fluxes only.
} 
as a strong criterium in de Sitter model building. We end with some concluding remarks in section [7. Some details are relegated to the appendices.

\section{The 4D and 10D picture}

In this section we explain two alternative ways of searching for dS vacua. First we discuss the reduction of the 10D type IIA theory on an SU(3)-structure manifold to four dimensions in subsection 2.1. This leads to a 4D effective theory with a scalar potential $V$. One can then ask whether minima of $V$ exist for which $V>0$. This approach is widely used in the literature but certain questions can be easily addressed if one works directly with the 10D equations of motion. We discuss this in subsection 2.2 where we also list some advantages of this approach.

\subsection{Dimensional reduction and effective $4 \mathrm{D}$ theories}

Before one can reduce the action from ten to four dimensions one has to decide which fields one keeps in the $4 \mathrm{D}$ theory. For compactifications on an SU(3)-holonomy manifold $M_{6}$ (i.e. when $M_{6}$ is a $C Y_{3}$ manifold) one normally expands the 10D fields in the cohomology of $M_{6}$. Allowing the coefficients in this expansion to depend on the four non-compact directions, they become 4D fields in the effective 4D theory upon integrating the $10 \mathrm{D}$ action over $M_{6}$. These fields are the lightest fields of an infinite tower of Kaluza-Klein (KK) fields that result from the compactification. Because these 4D scalar fields would be massless in the absence of fluxes, we will refer to them as moduli (even when now they have flux-induced masses). Restricting to these fields gives an effective 4D low-energy theory that is valid for energies below the KK-scale, which is approximately given by the inverse length of the cycles in $M_{6}$. Consistency requires that the flux-induced masses for the scalar fields are smaller than the KK-scale which should generically be true for sufficiently large $M_{6}$ [37.

When $M_{6}$ is a non-Ricci flat SU(3)-structure manifold, it is not clear anymore what the lightest fields are and how to obtain a low-energy effective action [38, 39. However, for the specific case of group manifolds we can restrict to expansion forms that are left-invariant under the group action. This leads to a $4 \mathrm{D}$ theory that is a consistent truncation [30, i.e. a solution to the $4 \mathrm{D}$ equations of motion will also be a solution to the $10 \mathrm{D}$ equations of motion. This should not change when one additionally does an orientifold projection. This was explicitly confirmed in [19] where it was shown that the 4D dS solution of [8] also solves the 10D equations of motion. We will therefore restrict ourselves to group spaces and expand the 10D fields and fluxes in left-invariant forms. Our conventions for the forms are given in appendix $\mathrm{A}$.

We now describe the 4D fields in the bosonic sector (the fermionic fields are determined by $\mathcal{N}=1$ supersymmetry). On an SU(3)-structure manifold $M_{6}$ there exists a real twoform $J=k^{i} Y_{i}^{(2-)}$ and a holomorphic three-form $\Omega$, which are generalization of the Kähler form and holomorphic three-form that exist on $C Y_{3}$ manifolds. The volume of $M_{6}$ in string 
frame is given by

$$
\operatorname{vol}_{6}=\frac{1}{6} \int J \wedge J \wedge J=\frac{1}{6} \kappa_{i j k} k^{i} k^{j} k^{k}
$$

and we use conventions in which $\Omega \wedge \Omega^{*}=\frac{4 i}{3} J \wedge J \wedge J$. In order to preserve only $\mathcal{N}=1$ supersymmetry in $4 \mathrm{D}$ we do an orientifold projection, which contains an anti-holomorphic involution $\sigma$. This leads to O6-planes that extend along the external four dimensions and wrap internal 3-cycles that are the fixed loci of $\sigma$. The action of $\sigma$ on $J$ and $\Omega$ is

$$
\sigma: \Omega \rightarrow-\Omega^{*}, \quad \sigma: J \rightarrow-J
$$

so that we can expand $\Omega=\Omega_{R}+i \Omega_{I}=\mathcal{F}_{K} Y^{(3-) K}+i \mathcal{Z}^{K} Y_{K}^{(3+)}$, where $\mathcal{F}_{K}$ and $\mathcal{Z}^{K}$ are real. Here the + and the - indicate the parity under the orientifold involution. For more notation conventions about our left-invariant forms see appendix A. Note that the $\mathcal{F}_{K}$ are functions of the $\mathcal{Z}^{K}$ and therefore not independent. The $\mathcal{Z}^{K}$ are projective coordinates so that only $h^{2,1}$ of them are independent.

In addition to these fields we have in type IIA supergravity the dilaton $\phi$, the KalbRamond two-form $B$ and the RR three-form $C_{3}$. Note that on a homogeneous manifold the $\mathrm{RR}$ form $C_{1}$ is not compatible with a strict $\mathrm{SU}(3)$-structure, which requires the absence of nowhere-vanishing one-forms. The $4 \mathrm{D}$ scalars arising from the reduction of these fields can be grouped into two sets of complex scalars

$$
\begin{aligned}
J_{c} & =J-i B=t^{i} Y_{i}^{(2-)}=\left(k^{i}-i b^{i}\right) Y_{i}^{(2-)} \\
\Omega_{c} & =\mathrm{e}^{-\phi} \operatorname{Im}(\Omega)+i C_{3}=N^{K} Y_{K}^{(3+)}=\left(u^{K}+i c^{K}\right) Y_{K}^{(3+)} .
\end{aligned}
$$

Note that $\mathrm{e}^{-\phi} \operatorname{Im}(\Omega)$ combines the dilaton with the $\mathcal{Z}^{K}$ so that all the $h^{2,1}+1$ of the $u^{K}$ are independent degrees of freedom.

The reduction of the $C_{3}$ field also leads to $4 \mathrm{D}$ vector fields

$$
C_{3}=A^{\alpha} Y_{\alpha}^{(2+)}
$$

so that we have the $4 \mathrm{D}$ gauge group $U(1)^{h_{+}^{1,1}}$. These are the only vector fields in the $4 \mathrm{D}$ theory since there are no one-forms that could lead to vector fields coming from the metric and $B$-field, and the $C_{1}$ field is odd under the orientifold projection so that it is projected out. Besides these scalar and vector fields and the 4D metric there are no further bosonic fields in the 4D theory.

Similarly to the fields we can expand the fluxes in terms of left-invariant forms which have the correct parity under the orientifold projection 6

$$
H=h_{K} Y^{(3-) K}, \quad \hat{F}_{0}=f_{(0)}, \quad \hat{F}_{2}=f_{(2)}^{i} Y_{i}^{(2-)}, \quad \hat{F}_{4}=f_{(4) i} Y^{(4+) i}, \quad \hat{F}_{6}=f_{(6)} Y^{(6-)} .
$$

\footnotetext{
${ }^{6}$ The only external flux that preserves $4 \mathrm{D}$ Lorentz symmetry is $F_{4}$-flux along the four space-time directions. We find it more convenient to work with its dual $\hat{F}_{6}$.
} 
For a general SU(3)-structure manifold the left-invariant forms are not necessarily in cohomology and therefore they are not necessarily closed under the exterior derivative $d$. However, d maps left-invariant forms into left-invariant forms so that we can write

$$
\mathrm{d} Y_{i}^{(2-)}=-r_{i K} Y^{(3-) K}, \quad \mathrm{~d} Y_{\alpha}^{(2+)}=-\hat{r}_{\alpha}{ }^{K} Y_{K}^{(3+)} .
$$

Since $\int \mathrm{d}\left(Y^{(2 \mp)} \wedge Y^{(3 \pm)}\right)=0$, this implies

$$
\mathrm{d} Y_{K}^{(3+)}=-r_{i K} Y^{(4+) i}, \quad \mathrm{~d} Y^{(3-) K}=\hat{r}_{\alpha}{ }^{K} Y^{(4-) \alpha} .
$$

The matrices $r_{i K}$ and $\hat{r}_{\alpha}{ }^{K}$ contain so called metric fluxes that are T-dual to $H$-flux. On group manifolds with $\mathrm{SU}(3)$-structure one always has six global left-invariant one-forms $e^{a}, a=1, \ldots, 6$ and one often defines the metric fluxes $f_{b c}^{a}$ through $\mathrm{d} e^{a}=-\frac{1}{2} f_{b c}^{a} e^{b} \wedge e^{c}$. The $r_{i K}$ and $\hat{r}_{\alpha}{ }^{K}$ are then linear functions of the $f_{b c}^{a}$.

The action of $\mathcal{N}=1$ supergravity in four dimensions 7 is determined by the real Kähler potential $K$, the holomorphic superpotential $W$ and the holomorphic gauge kinetic couplings $f_{\alpha \beta}$

$$
\begin{aligned}
S^{(4)}=-\int & {\left[-\frac{1}{2} R \star_{4} 1+K_{i \bar{\jmath}} \mathrm{d} t^{i} \wedge \star_{4} \mathrm{~d} \overline{t^{j}}+K_{K \bar{L}} \mathrm{~d} N^{K} \wedge \star_{4} \mathrm{~d} \overline{N^{L}}+V \star_{4} 1\right.} \\
+ & \left.\frac{1}{2} \operatorname{Re}\left(f_{\alpha \beta}\right) F^{\alpha} \wedge \star_{4} F^{\beta}+\frac{1}{2} \operatorname{Im}\left(f_{\alpha \beta}\right) F^{\alpha} \wedge F^{\beta}\right]
\end{aligned}
$$

where $K_{i \bar{\jmath}}=\partial_{t^{i}} \overline{\partial_{t^{j}}} K, K^{i \bar{\jmath}}$ is its transposed inverse and similarly for the $N^{K}$. The field strengths are $F^{\alpha}=\mathrm{d} A^{\alpha}$ and the $4 \mathrm{D}$ scalar potential is

$$
V=\mathrm{e}^{K}\left(K^{i \bar{\jmath}} \mathrm{D}_{t^{i}} W \overline{\mathrm{D}_{t^{j}} W}+K^{K \bar{L}} \mathrm{D}_{N^{K}} W \overline{\mathrm{D}_{N^{L}} W}-3|W|^{2}\right)+\frac{1}{2}(\operatorname{Re} f)^{-1^{\alpha \beta}} D_{\alpha} D_{\beta},
$$

where the derivatives $\mathrm{D}_{t^{i}} W=\partial_{t^{i}} W+W \partial_{t^{i}} K$ (and analogously for $\mathrm{D}_{N^{K}}$ ) should not be confused with the D-terms $D_{\alpha}$ which are given by

$$
D_{\alpha}=i\left(\delta_{\alpha} t^{i} \partial_{t^{i}} K+\delta_{\alpha} N^{K} \partial_{N^{K}} K\right)+i \frac{\delta_{\alpha} W}{W} .
$$

Here $\lambda^{\alpha} \delta_{\alpha} t^{i}$ is the variation of the field $t^{i}$ under the infinitesimal gauge transformation $A^{\alpha} \rightarrow A^{\alpha}+\mathrm{d} \lambda^{\alpha}$ and similarly for $N^{K}$ and $W$. Equation (2.10) is only valid for $W \neq 0$ but for the reductions we consider here (and generalizations thereof) one can show that $\delta_{\alpha} W$ always vanishes [40, 41. This means that the last term in (2.10) vanishes and equation (2.10) without the last term is true even for $W=0$.

\footnotetext{
${ }^{7}$ Except in section [6] and appendix [D we set $2 \pi \sqrt{\alpha^{\prime}}=1$. The pre-factor $\frac{1}{2 \kappa_{10}^{2}}=\frac{1}{(2 \pi)^{7}\left(\alpha^{\prime}\right)^{4}}=2 \pi$ in the usual 10D action can be removed by rescaling the action.
} 
The explicit forms of $K, W, f_{\alpha \beta}$ and $D_{\alpha}$ for the reduction of type IIA supergravity on $\mathrm{SU}(3)$-structure spaces have been derived in many papers [37,40,49] and the results are

$$
\begin{aligned}
K & =-2 \ln \left(-i \int \mathrm{e}^{-2 \phi} \Omega \wedge \Omega^{*}\right)-\ln \left(\frac{4}{3} \int J \wedge J \wedge J\right)=4 \phi_{4}-\ln \left(8 \mathrm{vol}_{6}\right), \\
\sqrt{2} W & =\int\left(\Omega_{c} \wedge\left(-i H+\mathrm{d} J_{c}\right)+\mathrm{e}^{i J_{c}} \wedge \hat{F}\right) \\
& =-N^{K}\left(i h_{K}+r_{i K} t^{i}\right)+f_{(6)}+i f_{(4) i} t^{i}-\frac{1}{2} \kappa_{i j k} f_{(2)}^{i} t^{j} t^{k}-\frac{i}{6} f_{(0)} \kappa_{i j k} t^{i} t^{j} t^{k}, \\
f_{\alpha \beta} & =-\hat{\kappa}_{i \alpha \beta} t^{i} \\
D_{\alpha} & =\frac{\mathrm{e}^{\phi_{4}}}{\sqrt{2 \mathrm{vol}_{6}}} \hat{r}_{\alpha}^{K} \mathcal{F}_{K},
\end{aligned}
$$

where $\phi_{4}$ is the $4 \mathrm{D}$ dilaton defined by $\mathrm{e}^{-\phi_{4}}=\mathrm{e}^{-\phi} \sqrt{\operatorname{vol}_{6}}$ and $\hat{F}=\hat{F}_{0}+\hat{F}_{2}+\hat{F}_{4}+\hat{F}_{6}$ is the sum of the RR fluxes.

There is one 10D equation of motion, the $\hat{F}_{2}$ Bianchi identity, that is not taken into account in this $4 \mathrm{D}$ analysis. It reads $\mathrm{d} \hat{F}_{2}+\hat{F}_{0} H=-j^{(\delta)}$, where $j^{(\delta)}$ is related to the Poincaré dual of the submanifold wrapped by the O6-planes (cf. section 4 and appendix D). In this paper we will follow the literature and only solve the integrated equation $-r_{i K} f_{(2)}^{i} Y^{(3-) K}+f_{(0)} h_{K} Y^{(3-) K}+j=0$, where the delta-functions in $j^{(\delta)}$ are replaced by one in $j 8$ The reason for this approximation is that, to our knowledge, there are no known solutions for (fully back-reacted) intersecting O-planes since the equations of motion with the delta-functions are very hard to solve.

For any given compactification space it is straightforward to solve the F- and D-term equations

$$
\mathrm{D}_{t^{i}} W=\mathrm{D}_{N^{K}} W=0, \quad D_{\alpha}=0,
$$

and to find supersymmetric AdS or Minkowski vacua. For AdS vacua it is generically possible to stabilize all moduli except when $\operatorname{rank}\left(r_{i K}\right)<h^{2,1}$ in which case $h^{2,1}-\operatorname{rank}\left(r_{i K}\right)$ $C_{3}$ axions remain flat directions [40. Note that this is not really a problem since the moduli space of the axions is compact. In supersymmetric Minkowski vacua it is not possible to stabilize all geometric moduli [40, 50].

Since all geometric moduli are stabilized in AdS vacua we know that the scalar potential depends on all geometric moduli and it is therefore sensible to ask whether the scalar potential allows for $\mathrm{dS}$ vacua as well. It is straightforward but tedious to calculate the explicit scalar potential (2.9) using (2.11) (see equation (2.34) in [9]). In order to find dS vacua one has to minimize this scalar potential with respect to all the moduli which is technically hard and can normally only be done numerically and only for SU(3)-structure manifolds with very few scalar fields. The authors of [51] scanned numerically the scalar potentials of the three models [37, 44, 48] for regions that allow for slow-roll inflation or dS vacua, but did not find any. This called for a different approach. In [4] the authors

\footnotetext{
${ }^{8}$ Since the integration essentially replaces delta-functions with 1 , solving integrated equations of motion is often called 'smearing the sources' in the literature.
} 
derived a no-go theorem that forbids slow-roll inflation and dS vacua in type IIA flux compactifications on $\mathrm{CY}_{3}$ manifolds with 06 -planes. However, this no-go theorem can be circumvented in compactifications on $\mathrm{SU}(3)$-structure manifolds that are not Ricci-flat, but instead negatively curved. This is the framework we will explore.

\subsection{The "universal" ansatz in $10 \mathrm{D}$}

$4 \mathrm{D}$ effective field theories correctly describe fluctuations around a string (or supergravity) solution, whenever the 4D solution can be lifted to a full solution of the 10D equations of motion, or at least to approximate solutions (in the sense described below). If every solution can be lifted to a full 10D solution, the reduction is said to be mathematically consistent. Even when this requirement is not satisfied, the reduction can still be physically consistent in the sense that while it does not fully describe the 10D physics, the effects of the heavy fields, the ones we ignore, are not relevant at low energies. This is what we meant by approximate 10D solutions. As an example, reductions on generic Calabi-Yau spaces have not proven to be mathematically consistent but are expected to be physically consistent when the internal volume is large enough such that we can ignore the KK modes. As explained above, for internal spaces with enough symmetries we expect dimensional reduction can be fully mathematically consistent if we restrict to the left-invariant modes and work in the limit that the sources are smeared. In that sense we should not be bothered with the question whether we are solving the 10D equations of motion, although we do not always have a separation of scales in our models. However as explained in [10, 19] it is interesting to work in the 10D picture because of several reasons: 1) Flux and charge quantization require us to know the 10D solution; 2) Computing the back-reaction of the sources, i.e. going beyond the smeared limit, is something that requires the 10D picture as well; 3) In ten dimensions one can establish solutions to the equations of motion without fully specifying the internal manifold.

Let us elaborate on point 3. Points 1 and 2 are discussed later in this paper. In order to find a de Sitter minimum in the $4 \mathrm{D}$ picture one has to minimize the scalar potential with respect to the moduli. This requires an explicit choice of manifold and that makes the procedure model dependent. On the other hand, when one solves 10D equations of motion it becomes clear that one can find solutions by just specifying properties of the internal manifold, but without fixing it entirely. This is for instance the case with SUSY AdS vacua in IIA from SU(3)-structure manifolds [52] (and also non-SUSY AdS vacua, see [53]). Therefore one constructs solutions for classes of manifolds that just obey the given properties. A downside of this approach is that it is not possible to compute the mass spectrum since that requires the explicit choice of the manifold. We will refer to such solutions as universal solutions. The idea of universal solutions was applied to the case of $\mathrm{dS}$ vacua in [10] and [19]. We will now review this approach and generalize it a bit.

So we start with an orientifold of a general SU(3)-structure manifold. As in the previous papers on the topic we make the simplification that the $\mathrm{SU}(3)$-structure is half-flat (see appendix $(\mathrm{B})$. In the $4 \mathrm{D}$ picture this restriction corresponds to the assumption that there are no non-zero D-terms (cf. appendix A of [40]). It would be interesting to extend this 
10D approach to manifolds that are not half-flat.

A half-flat manifold possesses a set of canonical forms, which we call universal forms and they are given by the (would-be) real Kähler form $J$ and the (would be) holomorphic complex three-form $\Omega$, and the torsion classes $W_{1}, W_{2}, W_{3}$

$$
\text { universal forms: } \quad\left\{J, \Omega, W_{1}, W_{2}, W_{3}\right\} \text {. }
$$

These then serve as natural expansion forms for the fluxes. Hence a general ansatz for a solution could be given by

$$
\begin{aligned}
e^{\Phi} \hat{F}_{0} & =f_{1}, \\
e^{\Phi} \hat{F}_{2} & =f_{2} J+f_{3} \hat{W}_{2}, \\
e^{\Phi} \hat{F}_{4} & =f_{4} J \wedge J+f_{5} \hat{W}_{2} \wedge J, \\
e^{\Phi} \hat{F}_{6} & =f_{6} \operatorname{vol}_{6}, \\
H & =f_{7} \Omega_{R}+f_{8} \hat{W}_{3}, \\
j & =j_{1} \Omega_{R}+j_{2} \hat{W}_{3} .
\end{aligned}
$$

where the fluxes are decomposed as follows:

$$
F=\hat{F}+\operatorname{vol}_{4} \wedge \tilde{F} .
$$

The fluxes $\hat{F}$ and $\tilde{F}$ have only components in the internal dimensions. We furthermore used the notation of [19] where $\hat{W}^{i}=\left(\sqrt{\left|W^{i}\right|^{2}}\right)^{-1} W^{i}$ 9. This ansatz is consistent with the orientifold involutions for supersymmetrically embedded orientifold planes [32. In order to check for which coefficients $f_{1}, \ldots, f_{8}$ and $j_{1}, j_{2}$ we have a solution we need the expression for the Ricci tensor as demanded by the Einstein equations. The Ricci tensor for a general $\mathrm{SU}(3)$-structure manifold has been established in [54 and is presented in appendix B. The relevant property is that it is given in terms of the universal forms. It is for this reason that a universal ansatz (where the fluxes and sources are given by universal forms) is sensible, since the Einstein equation forces the energy-momentum tensor to be made from universal forms. However, to our surprise, most de Sitter solutions in the models we consider below are not universal. This implies that there must be non-trivial cancelations of the non-universal flux pieces in the energy-momentum tensor.

It turns out that in order to find solutions different from the SUSY AdS solutions, one needs to impose constraints on the universal forms. These constraints are such that the equations of motion imply fewer constraints and therefore make possible the existence of

\footnotetext{
${ }^{9}$ We define the square of a $p$-form as $A_{p}^{2}=\frac{1}{p !} A_{i_{1} \ldots i_{p}} A^{i_{1} \ldots i_{p}}$.
} 
new solutions. These constraints are

$$
\begin{aligned}
\mathrm{d} \hat{W}_{2} & =c_{1} \Omega_{R}+d_{1} \hat{W}_{3}, \\
\hat{W}_{2} \wedge \hat{W}_{2} & =c_{2} J \wedge J+d_{2} \hat{W}_{2} \wedge J, \\
\mathrm{~d} \star_{6} \hat{W}_{3} & =c_{5} J \wedge J+c_{3} \hat{W}_{2} \wedge J, \\
\frac{1}{2}\left(\hat{W}_{3 i k l} \hat{W}_{3 j}{ }^{k l}\right)^{+} & =d_{4} J_{i k} \hat{W}_{2}{ }^{k}{ }_{j} .
\end{aligned}
$$

where

$$
\begin{aligned}
& c_{1}=-\frac{w_{2}}{4}, \quad c_{2}=-\frac{1}{3 !}, \quad c_{3}=-d_{1}, \quad c_{4}=\frac{1}{2}, \quad c_{5}=\frac{w_{3}}{3 !} \\
& d_{2}=-\star_{6}\left(\hat{W}_{2} \wedge \hat{W}_{2} \wedge \hat{W}_{2}\right),
\end{aligned}
$$

and

$$
w_{2}=\sqrt{W_{2}^{2}}, \quad w_{3}=\sqrt{W_{3}^{2}} .
$$

It is then straightforward to put the ansatz into the IIA equations of motion (see [19]) to obtain the algebraic equations for the flux parameters. These are very lengthy expressions and we therefore present them in appendix C. It is very non-trivial to find the general solution to these algebraic equations but many solutions have nonetheless been found.

Let us review these solutions

- The SUSY AdS solutions necessarily have $W_{3}=0$ and they require us to enforce the first constraint in equations (2.16) [52,55].

- Non-SUSY AdS solutions can be found when $W_{3}=0$ when we also enforce the second constraint in equations (2.16) $[10,53]$.

- De Sitter solutions can be found under the same circumstances as the above nonSUSY AdS vacua [10], however no explicit geometry has been found that satisfies the parameter windows that gives these dS solutions, as opposed to the AdS solutions.

- Universal solutions with non-zero $W_{3}$ have been investigated in [19] but with the simplification that $W_{2}=0$. In that case AdS, Minkowski and dS solutions are possible when we enforce the third and fourth constraint in equations (2.16) with the choice $d_{4}=0$. In fact one extra constraint was necessary, namely $Q_{1}\left(\hat{W}_{3}, \hat{W}_{3}\right) \propto$ $Q_{2}\left(\hat{W}_{3}, \hat{W}_{3}\right) \propto\left(\hat{W}_{3}\right)_{2,1}$, where we refer to appendix $\mathrm{B}$ for the definitions of $Q_{1}$ and $Q_{2}$. Interestingly, there exists at least one explicit geometry that satisfies the conditions for these universal dS solutions, namely $\mathrm{SU}(2) \times \mathrm{SU}(2)$ as was shown in [19.

\section{Classification of geometries}

\subsection{Homogenous SU(3)-structures}

We want to classify homogeneous geometries that are consistent with an SU(3)-structure that is invariant under the left acting isometries. The covering space of a homogenous 
manifold is necessarily a coset $G / H$ (or a group when $H=1$ ). Furthermore one can show that $H \subseteq \mathrm{SU}(3)$ if we want the coset to allow an invariant $\mathrm{SU}(3)$-structure [55]10.

The requirement of homogeneity and an invariant SU(3)-structure are strong constraints and the problem of classifying the spaces turns into an algebraic problem that involves two steps. Firstly, one has to use group theory to classify the possible covering spaces $G / H$. Secondly, one has to classify the possible discrete subgroups $L \subset G$ that one can use to create another space $G / H / L$. For instance, when the covering space is non-compact we need to find a discrete subgroup that can render the manifold compact after division. Furthermore we need discrete subgroups that contain involutions for the orientifold action.

In figure 1 we sketch the classification of (the covering spaces of) homogeneous SU(3)structure manifolds.

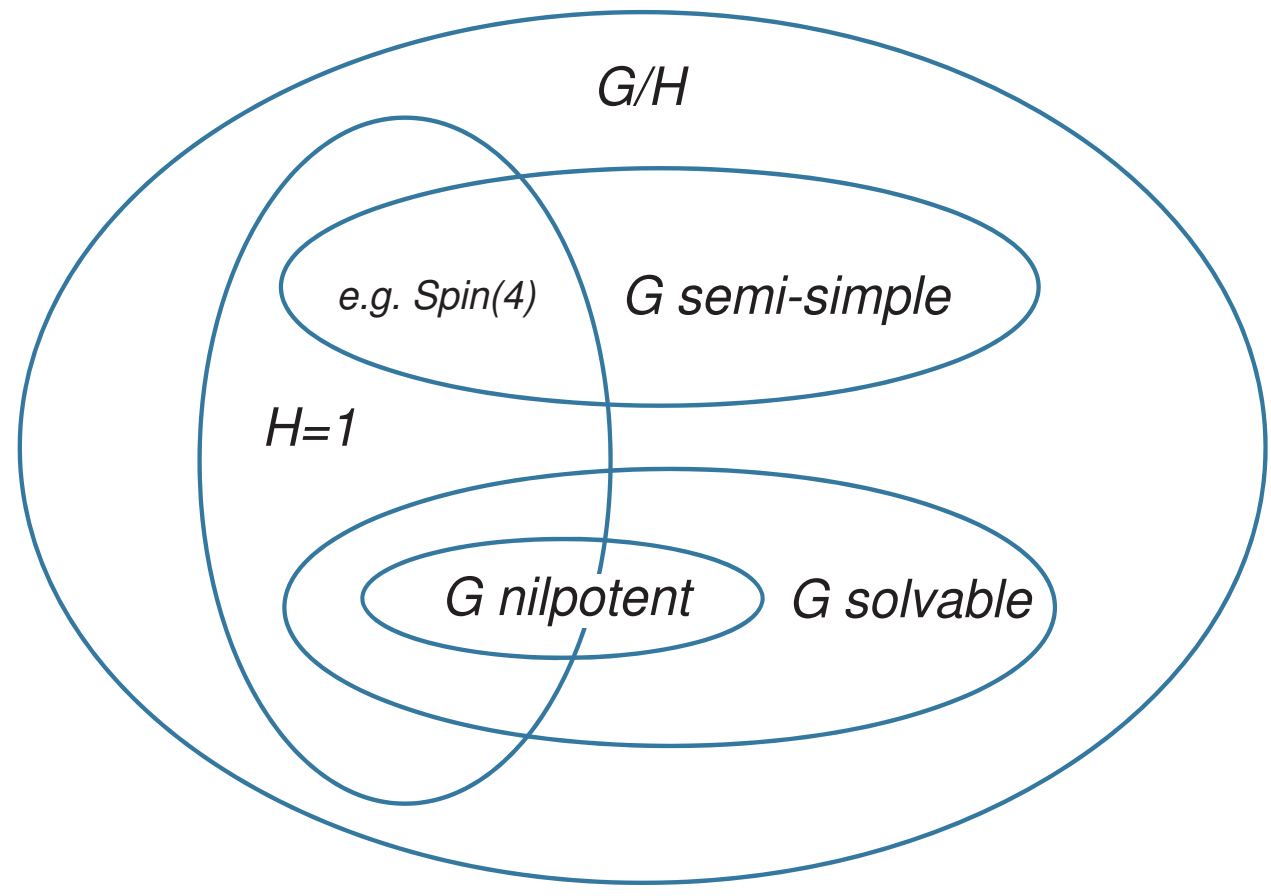

Figure 1: The homogeneous $\mathrm{SU}(3)$-structure spaces $G / H$. The isotropy group is necessarily contained in $\mathrm{SU}(3)$ for a $G$-invariant $\mathrm{SU}(3)$-structure.

The picture shows the separate subset of cosets that are group spaces $(H=\{1\})$. 11 Lie groups can be classified using the properties of the associated Lie algebra that can range

\footnotetext{
${ }^{10}$ If $H$ is not within $\mathrm{SU}(3)$ there could still be other non-G-invariant $\mathrm{SU}(3)$-structures, but they are outside this particular coset description. It could be (but by no means necessarily so) that they are described by another coset description, because sometimes the same manifold can have different coset descriptions, e.g. $\mathbb{C P}^{3}=\mathrm{Sp}(2) / S(\mathrm{U}(2) \times \mathrm{U}(1))=\mathrm{SU}(4) / S(\mathrm{U}(3) \times \mathrm{U}(1))$. In this case there is a $\mathrm{Sp}(2)$ invariant homogeneous $\mathrm{SU}(3)$-structure, but no $\mathrm{SU}(4)$-invariant one.

${ }^{11}$ Groups and cosets are a trivial kind of $\mathrm{SU}(3)$-structure since the structure group of the frame bundle is trivial. In this paper we consider orbifolds therefor such that the frame bundle is not trivial anymore and we have a genuine SU(3)-structure, after blowing up the orbifold singularities.
} 
from being semi-simple to the opposite, being nilpotent. The solvable cases are somewhere in between. Especially the nilpotent examples have received much attention since they are the covering space of compact spaces obtained from T-duality of a torus with $H$-flux. They are called twisted tori. But the name twisted tori is sometimes also given to the solvable cases and even all group spaces.

Let us discuss those cases that have already made an appearance in the literature. Reference [55] classified those cosets that have an isometry group $G$ which is a compact semi-simple group or the product thereof with U(1)-factors. This also includes the space $\operatorname{Spin}(4)=\mathrm{SU}(2) \times \mathrm{SU}(2)$, which we have depicted in the intersection of groups and cosets with semi-simple isometry groups.

The class of cosets in 55] is not the most general class of cosets with non-trivial isotropy because one can consider spaces with non-compact $G$ or with $G$ generically non-semisimple. Examples of this sort have not appeared in the literature to our knowledge. We will not deal with those examples here, but they might offer an interesting class of coset geometries to investigate de Sitter solutions. Reference [9] considered all possible "metric fluxes" consistent with the symmetries of all the abelian orbifold groups of the six-torus. However they did not provide any further description of these spaces like for example the Lie algebra of symmetries. In contrast reference $[56]^{12}$ made a less complete list (solvmanifolds and nilmanifolds), but has a partial description of the geometries. Below we present a full (and simple) classification of covering spaces $G$ using group theory, which allows us to have an algebraic understanding of the various metric fluxes. We also find more possibilities for orientifolds than the ones given in [56].

\subsection{Group manifold geometry and geometric moduli}

We recall the basic concepts of the geometry of a group manifold. Although this has been done in many places before, we repeat this here such that the paper is self-contained.

On a group manifold, $G$, one can define a co-frame of left-invariant forms, called the Maurer-Cartan forms, as follows,

$$
g^{-1} \mathrm{~d} g=e^{a} T_{a},
$$

where the $T^{a}$ are the generators of the Lie algebra associated to $G$ and denoted $\mathfrak{g}$. The Maurer-Cartan forms obey the relations

$$
\mathrm{d} e^{a}=-\frac{1}{2} f_{b c}^{a} e^{b} \wedge e^{c},
$$

where the $f^{\prime}$ 's are the structure constants of $\mathfrak{g}$. The conditions for a set of $f^{a} b c$ to describe a Lie algebra are

$$
f_{b c}^{a}=-f_{c b}^{a}, \quad f_{b[c}^{a} f_{d e]}^{b}=0 .
$$

\footnotetext{
${ }^{12}$ Later this has been significantly been improved in 17 .
} 
It can be shown that only unipotent groups, i.e. groups with traceless structure constants,

$$
f_{a b}^{a}=0, \quad \text { for all } b \text { (sum over } a \text { implied), }
$$

can be made compact. To be more precise, only unipotent groups $G$ can have a discrete subgroup, $L$, acting without fixed points, such that $G / L$ is a compact space. If the group is already compact $L$ can of course taken to be trivial. Unipotence, however, is not a sufficient condition to establish the existence of such a discrete subgroup, see e.g. [17, 56] and references therein.

In terms of the Maurer-Cartan one-forms, $e^{a}=e_{\mu}^{a} \mathrm{~d} x^{\mu}$, we can introduce a metric on the group manifold,

$$
\mathrm{d} s^{2}=\mathcal{M}_{a b} e^{a} \otimes e^{b},
$$

where $\mathcal{M}$ is constant, symmetric and positive definite. The Ricci tensor is then given by (see e.g. [57])

$$
R_{a b}=\frac{1}{4} f_{a c d} f_{b}^{c d}-\frac{1}{2} f_{d a}^{c} f_{c b}^{d}-\frac{1}{2} f_{c d a} f_{b}^{c d},
$$

where we lower and raise indices using the metric $\mathcal{M}$ and its inverse. Furthermore we restricted to algebras for which $f_{a b}^{a}=0$, (sum over $a$ implied), since we are not interested in non-compact models. The Ricci scalar then reads

$$
R=-\frac{1}{4} f_{a b c} f^{a b c}-\frac{1}{2} f_{c a b} f^{a c b} .
$$

The matrix $\mathcal{M}$ parameterizes that part of the group manifold moduli space that is concerned with metric deviations along left-invariant directions. This moduli space is $\mathrm{GL}(n, \mathbb{R}) / \mathrm{SO}(n)$, as can be seen from the fact that the matrix $\mathcal{M}$ transforms under $G L(n, \mathbb{R})$-matrices, but is invariant under an $\mathrm{SO}(n)$-group. When we consider orbifold and orientifold symmetries we put further restrictions on $\mathcal{M}$ such that the geometric moduli space gets truncated.

\subsection{All unipotent real six-dimensional Lie algebras}

Here, we would like to classify all six-dimensional real Lie algebras. The classification is already done in the literature [58], but in our case the classification is simplified because we restrict to unipotent algebras.

Lie algebras are classified depending on whether or not they have ideals. We remind ourselves that an ideal $\mathfrak{i}$ of an algebra $\mathfrak{g}$ is a subalgebra with the following property: $[\mathfrak{g}, \mathfrak{i}] \subseteq \mathfrak{i}$. On the one end of the collection of Lie algebras one has simple Lie algebras. They are defined as the Lie algebras that have no proper ideal. Next to those come the semi-simple Lie algebras which have no proper abelian ideals. One can show that they are direct sums of simple Lie algebras. The definition of a semi-simple Lie algebra turns out to be equivalent to having a non-degenerate Killing form $C$, where $C_{a b}=f_{d a}^{c} f_{c b}^{d}$. On the other end of the spectrum of Lie algebras are those that have big ideals. One way to built ideals is by taking commutators. Consider the following sets of subalgebras of $\mathfrak{g}$

$$
\mathfrak{g}^{n}=\left[\mathfrak{g}^{n-1}, \mathfrak{g}^{n-1}\right], \quad \mathfrak{g}_{n}=\left[\mathfrak{g}, \mathfrak{g}_{n-1}\right],
$$


where $\mathfrak{g}^{0}=\mathfrak{g}_{0}=\mathfrak{g}$. Both the upper-derived series, $\mathfrak{g}^{n}$, and the lower-derived series, $\mathfrak{g}_{n}$, are obviously ideals. A solvable Lie algebra is a Lie algebra for which the upper-derived series vanishes at some point and for a nilpotent Lie algebra the lower-derived series vanishes at some point. It is easy to show that $\mathfrak{g}^{n} \subseteq \mathfrak{g}_{n}$, from which follows that a nilpotent algebra is also solvable. One can prove that the nilpotent Lie algebras form the building blocks for the solvable algebras by taking semi-direct products, whereas the solvable and simple Lie algebras are the building blocks for all remaining Lie algebras, again by taking semi-direct products. The latter is the content of Levi's theorem, which states that any Lie algebra $\mathfrak{g}$ is the semi-direct product of a semi-simple algebra $\mathfrak{s}$ with the largest solvable ideal $\mathfrak{r}$

$$
\mathfrak{g}=\mathfrak{s} \ltimes \mathfrak{r} .
$$

The largest solvable ideal, $\mathfrak{r}$, can be shown to be unique and is called the radical. Using this theorem the problem boils down to classifying semi-simple and solvable algebras of dimension $d \leq 6$ and the possible semi-direct products between the $m$-dimensional semisimple algebra $\mathfrak{s}$ and the $n$-dimensional radical $\mathfrak{r}$, where $m+n=6$.

Semi-simple and nilpotent algebras are always unipotent, but unipotence has to be verified for solvable groups. Note that for a general Lie algebra to be unipotent, the radical itself has to be unipotent. Indeed, using indices $a^{\prime}, b^{\prime}$ to run over $\mathfrak{s}$ and $a^{\prime \prime}, b^{\prime \prime}$ to run over $\mathfrak{r}$ we find

$$
0=f_{a b^{\prime \prime}}^{a}=f_{a^{\prime} b^{\prime \prime}}^{a^{\prime}}+f_{a^{\prime \prime} b^{\prime \prime}}^{a^{\prime \prime}}=f_{a^{\prime \prime} b^{\prime \prime}}^{a^{\prime \prime}}
$$

since all $f_{a^{\prime} b^{\prime \prime}}^{a^{\prime}}=0$, as $\mathfrak{r}$ is an ideal.

The classification of the semi-simple algebras of dimension $d \leq 6$ is straightforward and one can find six examples

- $\mathfrak{s o}(p, q)$ with $p+q=4$.

- $\mathfrak{s o}(3) \times \mathfrak{s o}(2,1)$.

- $\mathfrak{s o}(p, q)$ with $p+q=3$.

Note that $\mathfrak{s o}(4)=\mathfrak{s o}(3)^{2}$ and $\mathfrak{s o}(2,2)=\mathfrak{s o}(2,1)^{2}$. So, there are four six-dimensional semisimple algebras and only the compact case $\mathfrak{s o}(4)$ has so far been used in the flux literature. For the other three cases, it is not clear whether there are points in the moduli space of these groups that allow a discrete subgroup $L$ that makes $G / L$ a smooth compact space.

To find the other six-dimensional group spaces using Levi's theorem we deduce that we need to know all six- and three-dimensional solvable algebras. The six-dimensional unipotent solvable algebras are the ones that describe the solvmanifolds and their classification appears in e.g. [56 [3. We will not repeat those algebras here but refer to tables 4 and 5 in [56]). The nilpotent ones have the nice feature that the associated group space $G$ always allows a discrete subgroup $L$ such that $G / L$ is a smooth compact space, called nilmanifolds.

\footnotetext{
${ }^{13}$ The classification of solvable algebras in [56] is not complete. There are some non-algebraic examples that are not given. For a complete list we refer to [59] or [17].
} 
Let us now focus on the remaining algebras that have a three-dimensional radical. This implies we have to find all solvable unipotent three-dimensional Lie algebras first. This is straightforward since they are contained in the list of six-dimensional unipotent solvable algebras as those which have a $\mathfrak{u}(1)^{3}$-factor. Alternatively one can simply use the known classification of three-dimensional Lie algebras (a.k.a. the Bianchi classification, see e.g. [60]) and check which are unipotent and solvable. There are only four of these and given explicitly by

- $\mathfrak{u}(1)^{3}$,

- Heisenberg: $\quad\left[r_{1}, r_{2}\right]=r_{3}$.

- $\mathfrak{i s o}(2): \quad\left[r_{1}, r_{2}\right]=r_{3}, \quad\left[r_{3}, r_{1}\right]=r_{2}$,

- $\mathfrak{i s o}(1,1): \quad\left[r_{1}, r_{2}\right]=r_{3}, \quad\left[r_{3}, r_{1}\right]=-r_{2}$,

where $r_{1}, r_{2}, r_{3}$ represent the generators and we only presented the non-zero commutators.

The next step is to consider the possible semi-direct products of these four solvable algebras with the two three-dimensional simple algebras $\mathfrak{s o}(3)$ and $\mathfrak{s o}(2,1)$. Let us recall the definition of a semi-direct product: a semi-direct product $\mathfrak{s} \ltimes \mathfrak{r}$ is the direct product as vector spaces equipped with a Lie bracket

$$
\left[\left(s_{1}, r_{1}\right),\left(s_{2}, r_{2}\right)\right]_{\mathfrak{s} \ltimes \mathfrak{r}}=\left(\left[s_{1}, s_{2}\right]_{\mathfrak{s}},\left[r_{1}, r_{2}\right]_{\mathfrak{r}}+\rho\left(s_{1}\right) r_{2}-\rho\left(s_{2}\right) r_{1}\right),
$$

for all $s_{1}, s_{2} \in \mathfrak{s}$ and $r_{1}, r_{2} \in \mathfrak{r}$. Here $\rho$ is a Lie algebra homomorphism of $\mathfrak{s}$ into the derivative operators on $\mathfrak{r}$. To be explicit, this means

$$
\begin{aligned}
\rho\left(\left[s_{1}, s_{2}\right]\right) & =\rho\left(s_{1}\right) \rho\left(s_{2}\right)-\rho\left(s_{2}\right) \rho\left(s_{1}\right) \quad \text { (Lie-algebra homomorphism), } \\
\rho(s)\left(\left[r_{1}, r_{2}\right]\right) & =\left[\rho(s)\left(r_{1}\right), r_{2}\right]+\left[r_{1}, \rho(s)\left(r_{2}\right)\right] \quad \text { (derivative operator). }
\end{aligned}
$$

Both properties are needed to assure the Jacobi identity of the semi-direct product. Since $\rho$ is a representation of $\mathfrak{s}$ in $\mathfrak{r}$ it should be possible to classify the different semi-direct products $\rho$ by the representation theory of $\mathfrak{s}$.

The possible representations of $\mathfrak{s o}(3)$ and $\mathfrak{s o}(2,1)$ must be three-dimensional. So we have

$$
\mathbf{1} \oplus \mathbf{1} \oplus \mathbf{1}, \quad \mathbf{1} \oplus \mathbf{2}, \quad \mathbf{3} .
$$

The representation $\mathbf{3}$ for $\mathfrak{s o}(3)$ is

$$
M_{1}=\left(\begin{array}{ccc}
0 & 1 & 0 \\
-1 & 0 & 0 \\
0 & 0 & 0
\end{array}\right), \quad M_{2}=\left(\begin{array}{ccc}
0 & 0 & 1 \\
0 & 0 & 0 \\
-1 & 0 & 0
\end{array}\right), \quad M_{3}=\left(\begin{array}{ccc}
0 & 0 & 0 \\
0 & 0 & 1 \\
0 & -1 & 0
\end{array}\right) .
$$

The representation $\mathbf{3}$ for $\mathfrak{s o}(2,1)$ is

$$
M_{1}=\left(\begin{array}{lll}
0 & 1 & 0 \\
1 & 0 & 0 \\
0 & 0 & 0
\end{array}\right), \quad M_{2}=\left(\begin{array}{lll}
0 & 0 & 1 \\
0 & 0 & 0 \\
1 & 0 & 0
\end{array}\right), \quad M_{3}=\left(\begin{array}{ccc}
0 & 0 & 0 \\
0 & 0 & 1 \\
0 & -1 & 0
\end{array}\right) .
$$


The representation $\mathbf{2}$ for $\mathfrak{s o}(3)$ is given by the Pauli matrices

$$
M_{1}=\left(\begin{array}{cc}
0 & 1 \\
1 & 0
\end{array}\right), \quad M_{2}=\left(\begin{array}{cc}
0 & i \\
-i & 0
\end{array}\right), \quad M_{3}=\left(\begin{array}{cc}
1 & 0 \\
0 & -1
\end{array}\right) .
$$

Since the Pauli matrices contain at least one complex generator (in any basis) we cannot build a real six-dimensional Lie-algebra from this representation of the semi-direct product. So the $\mathbf{1} \oplus \mathbf{2}$ is discarded for $\mathfrak{s}=\mathfrak{s o}(3)$. Through the Weyl unitary trick we find the twodimensional representation of $\mathfrak{s o}(2,1)$ to be real:

$$
M_{1}=\left(\begin{array}{ll}
0 & 1 \\
1 & 0
\end{array}\right), \quad M_{2}=\left(\begin{array}{cc}
0 & 1 \\
-1 & 0
\end{array}\right), \quad M_{3}=\left(\begin{array}{cc}
1 & 0 \\
0 & -1
\end{array}\right) .
$$

Hence, the $\mathbf{1} \oplus \mathbf{2}$ is still possible for $\mathfrak{s}=\mathfrak{s o}(2,1)$.

Having recalled the various representations we need to go from the representation $\rho$ to the construction of the six-dimensional Lie algebra, that is, the construction of the structure constants. For that, consider the bracket

$$
\left[s_{a}, r_{i}\right]=f^{j}{ }_{a i} r_{j} \equiv\left[M_{a}\right]_{i}^{j} r_{j},
$$

The three matrices $M_{a}$ make up the representation of the simple part $\mathfrak{s}=(\mathfrak{s o}(3)$ or $\mathfrak{s}=$ $\mathfrak{s o}(2,1))$.

For the 1 representation we have $M_{a}=0$, hence the six-dimensional algebra is the direct product of the solvable and the simple part. Further constraints come from the demand that $\rho$ is a derivation when $\rho$ is non-trivial (i.e. $\rho \neq \mathbf{1} \oplus \mathbf{1} \oplus \mathbf{1}$ ). We analyze this below, where we denote by $\rho$ the representation of an arbitrary element of $\mathfrak{s}$, that is $\rho=\rho\left(s_{i}\right)$ with $s_{i}$ arbitrary. So when we write $\rho\left(r_{1}\right)$ we really mean $\rho\left(s_{i}\right)\left(r_{1}\right)$. In components we write

$$
\rho\left(r^{i}\right)=\rho_{j}^{i} r^{j} .
$$

In what follows we investigate the conditions on the matrix elements $\rho_{j}^{i}$ coming from $(3.12 b)$.

- $\mathfrak{r}=\mathfrak{u}(1)^{3}$ : There are no conditions coming from (3.12b).

- $\mathfrak{r}=$ Heis $_{3}: \rho_{2}^{3}=\rho_{1}^{3}=0, \rho_{3}^{3}=\rho_{2}^{2}+\rho_{1}^{1}$. Hence only the $\mathbf{3}$ representation is excluded. For the $\mathbf{1} \oplus \mathbf{2}$ representation, $r_{3}$ is the singlet.

- $\mathfrak{r}=\mathfrak{i s o}(2) \& \mathfrak{i s o}(1,1): \rho_{1}^{3}=\rho_{1}^{2}=\rho_{1}^{1}=0, \quad \rho_{3}^{3}=\rho_{2}^{2}, \quad \rho_{3}^{2}=\mp \rho_{2}^{3}$, where the upper (lower) sign is for $\mathfrak{i s o}(2)(\mathfrak{i s o}(1,1))$. Both the $\mathbf{1} \oplus \mathbf{2}$ and the $\mathbf{3}$ representation are excluded.

Let us summarize by listing all unipotent algebras that we have found that are not solvable. First we have the four semi-simple cases, which we list in table 1.

Then we have the non-semi-simple, non-solvable unipotent examples, which we list in table 2 . 


\begin{tabular}{c}
\hline \hline Case \\
\hline $\mathfrak{s o}(3) \times \mathfrak{s o}(3)$ \\
$\mathfrak{s o}(3) \times \mathfrak{s o}(2,1)$ \\
$\mathfrak{s o}(2,1) \times \mathfrak{s o}(2,1)$ \\
$\mathfrak{s o}(3,1)$ \\
\hline
\end{tabular}

Table 1: The semi-simple six-dimensional Lie algebras

\begin{tabular}{cc}
\hline \hline Case & Representations \\
\hline $\mathfrak{s o}(3) \ltimes_{\rho} \mathfrak{u}(1)^{3}$ & $\rho=\mathbf{1} \oplus \mathbf{1} \oplus \mathbf{1}$ and $\rho=\mathbf{3}$ \\
$\mathfrak{s o}(3) \ltimes_{\rho} \mathrm{Heis}_{3}$ & $\rho=\mathbf{1} \oplus \mathbf{1} \oplus \mathbf{1}$ \\
$\mathfrak{s o}(3) \ltimes_{\rho} \mathfrak{i} \mathfrak{i o}(2)$ & $\rho=\mathbf{1} \oplus \mathbf{1} \oplus \mathbf{1}$ \\
$\mathfrak{s o}(3) \ltimes_{\rho} \mathfrak{i} \mathfrak{i o}(1,1)$ & $\rho=\mathbf{1} \oplus \mathbf{1} \oplus \mathbf{1}$ \\
$\mathfrak{s o}(2,1) \ltimes_{\rho} \mathfrak{u}(1)^{3}$ & $\rho=\mathbf{1} \oplus \mathbf{1} \oplus \mathbf{1}, \rho=\mathbf{1} \oplus \mathbf{2}$ and $\rho=\mathbf{3}$ \\
$\mathfrak{s o}(2,1) \ltimes_{\rho} \mathrm{Heis}_{3}$ & $\rho=\mathbf{1} \oplus \mathbf{1} \oplus \mathbf{1}$ and $\rho=\mathbf{1} \oplus \mathbf{2}$ \\
$\mathfrak{s o}(2,1) \ltimes_{\rho} \mathfrak{i} \mathfrak{i o}(2)$ & $\rho=\mathbf{1} \oplus \mathbf{1} \oplus \mathbf{1}$ \\
$\mathfrak{s o}(2,1) \ltimes_{\rho} \mathfrak{i s o}(1,1)$ & $\rho=\mathbf{1} \oplus \mathbf{1} \oplus \mathbf{1}$ \\
\hline
\end{tabular}

Table 2: The unipotent non-solvable, non-semi-simple six-dimensional Lie algebras

Hence there are 16 unipotent non-solvable six-dimensional Lie algebras. These have to be added to the list of solvable unipotent Lie algebras in [17,56]. As we mentioned before, unipotence is just one condition for a compactification $L$ to exist when the group $G$ is non-compact, but we do not know which of these examples cannot be made compact. The non-compact semi-simple cases of table 1 could be problematic. When the representation is trivial, $\rho=\mathbf{1} \oplus \mathbf{1} \oplus \mathbf{1}$, for the cases in table 2 and the simple part is $\mathfrak{s o}(3)$ then we know for sure that the space can be made compact since $\mathrm{SO}(3)$ is compact and the three-dimensional solvable groups can be made compact, see e.g. [7, version 1].

\section{Discrete symmetries, orbifolds and orientifolds}

\subsection{Discrete subgroups of SU(3)}

In this paper we restrict ourselves to the study of compactifications that preserve $\mathcal{N}=1$ in four dimensions since these are interesting for phenomenological reasons. Since group manifolds have a trivial structure group they lead after an orientifold projection to 4D theories with $\mathcal{N}=4$. Therefore we have to mod out the group manifolds by a discrete subgroup of $\mathrm{SU}(3)$ to obtain an $\mathcal{N}=1$ theory. Any dS critical point we find is then also a dS critical point of the parent $\mathcal{N}=4$ theory. General studies of $4 \mathrm{D} \mathcal{N}=4$ theories [18, 22, 23, 61 64] indicate that they do not allow for a metastable dS solution. However, when we truncate to an $\mathcal{N}=1$ theory we can hope that we project out the tachyonic directions and find stable $\mathrm{dS}$ vacua. An unstable $\mathrm{dS}$ extremum in the parent 
$\mathcal{N}=4$ theory could then be stable in the truncated $\mathcal{N}=1$ theory 14 . On general grounds one might expect that lowering the amount of supersymmetry of the effective action also lowers the constraints on obtaining stable $\mathrm{dS}$ critical points. This has been explicitly confirmed in [65] by the construction of stable dS solutions in $\mathcal{N}=2$ gauged supergravity. It is desirable to understand the higher-dimensional origin of this solution, as it is unknown as yet.

Let us now explain how we proceed to construct an orientifold to get an $\mathcal{N}=1$ supergravity. We can define three complex coordinates $z^{i}$ on the group spaces. In order to find an $\mathcal{N}=1$ theory we have to first act on these three complex coordinates (holomorphically) with a discrete subgroup $\Gamma \subset \mathrm{SU}(3)(\Gamma \nsubseteq \mathrm{SU}(2))$. Through its action on the $z^{i}, \Gamma$ also acts on the structure constants and it is severely constrained by the requirement that these are invariant. In the case that we have to mod out the group manifold by a lattice $L$ to make it compact, we also have to ensure that $\Gamma$ maps the lattice points (i.e. points in $G$ that are identified) into each other. This generically puts strong constraints on the possible $\Gamma$ for any compact $G / L$. In addition to this orbifold projection we do an orientifold projection that leads to O6-planes. The resulting space is the quotient $G / \Gamma / \hat{\sigma}$ with $\hat{\sigma}=\sigma \Omega_{p}(-1)^{F_{L}}$, where $\Omega_{p}$ is the world-sheet parity operator and $F_{L}$ is the space-time fermion number in the left-moving sector. Furthermore, we have to demand that $\hat{\sigma}$ is a $\mathbb{Z}_{2}$ extension of $\Gamma$ i.e. that for all $\gamma \in \Gamma$ we have $\gamma \hat{\sigma} \gamma \hat{\sigma} \in \Gamma$. In order to preserve supersymmetry $\sigma$ has to act anti-holomorphically on the $z^{i}$. The 3-cycles that are fixed under the action of $\sigma$ are wrapped by O6-planes.

While it is beyond the scope of this paper to list all possible orbifold and orientifold projections for all group spaces, we will briefly discuss all discrete subgroups of $\mathrm{SU}(3)$ [66 68 that are not contained in SU(2). There are two infinite series of abelian subgroups. The $\mathbb{Z}_{N}$ subgroups are generated by the following action

$$
\theta:\left(z^{1}, z^{2}, z^{3}\right) \rightarrow\left(\mathrm{e}^{2 \pi i n_{1} / N} z^{1}, \mathrm{e}^{2 \pi i n_{2} / N} z^{2}, \mathrm{e}^{2 \pi i\left(N-n_{1}-n_{2}\right) / N} z^{3}\right), \quad n_{1} \leq n_{2} \leq N-n_{1}-n_{2} .
$$

One can also combine two cyclic subgroups of $\mathrm{SU}(2)$ to get the subgroup $\mathbb{Z}_{N} \times \mathbb{Z}_{M}$ which is generated by

$$
\begin{aligned}
& \theta_{1}:\left(z^{1}, z^{2}, z^{3}\right) \rightarrow\left(\mathrm{e}^{2 \pi i / N} z^{1}, \mathrm{e}^{-2 \pi i / N} z^{2}, z^{3}\right), \\
& \theta_{2}:\left(z^{1}, z^{2}, z^{3}\right) \rightarrow\left(\mathrm{e}^{2 \pi i / M} z^{1}, z^{2}, \mathrm{e}^{-2 \pi i / M} z^{3}\right) .
\end{aligned}
$$

There are also two infinite series of non-abelian discrete subgroups called $\Delta\left(3 n^{2}\right)$ and $\Delta\left(6 n^{2}\right)$. Both of these groups have an abelian $\mathbb{Z}_{n} \times \mathbb{Z}_{n}$ subgroup. $\Delta\left(3 n^{2}\right)$ is an $\mathbb{Z}_{3}$ extension of this abelian group where the $\mathbb{Z}_{3}$ is generated by the following action

$$
\left(z^{1}, z^{2}, z^{3}\right) \rightarrow\left(z^{2}, z^{3}, z^{1}\right) .
$$

\footnotetext{
${ }^{14}$ Since we restrict ourselves to $\mathcal{N}=1$ theories we cannot exclude the possibility that one of our group manifolds leads to (un)stable dS solutions in $\mathcal{N}=2,4$ SUGRA that are not consistent with our orbifold projection i.e. that we do not find. It would therefore be very interesting to study compactifications on group spaces that lead to $\mathcal{N}=2,4$ theories.
} 
$\Delta\left(6 n^{2}\right)$ is an $S_{3}$ extension of $\mathbb{Z}_{n} \times \mathbb{Z}_{n}$, where $S_{3}$ is the permutation group of three elements that is of order 6 and acts on the three $z^{i}$. Finally there are the so called exceptional discrete subgroups of SU(3): $\Sigma_{36 \cdot \varphi}, \Sigma_{60 \cdot \varphi}, \Sigma_{72 \cdot \varphi}, \Sigma_{168 \cdot \varphi}, \Sigma_{216 \cdot \varphi}, \Sigma_{360 \cdot \varphi}$ with $\varphi=1,3$, where the subscript for $\varphi=1$ is the order of the group.

If $\Gamma$ has fixed points then $G / \Gamma$ has orbifold singularities at these fixed point 15 . This is sensible in string theory where a set of new degrees of freedom, called the twisted sector, arises at each orbifold singularity. In order to trust the supergravity analysis one has to resolve the singularity by "blowing it up". This leads to new cycles at the resolved orbifold singularity. These new cycles and their dual forms lead to new moduli but also allow us to turn on additional fluxes. We will not investigate these extra moduli and fluxes and expect that the extra moduli can be stabilized by the extra fluxes at a higher scale so that their impact on our analysis is small. This was explicitly confirmed for a concrete $C Y_{3}$ compactification in [37].

We will now illustrate the general discussion above for the explicit example of the $\mathbb{Z}_{2} \times \mathbb{Z}_{2}$ orbifold since it plays a prominent role in the construction of dS vacua.

\subsection{The standard $\mathbb{Z}_{2} \times \mathbb{Z}_{2}$ orientifold example}

\section{The classification of compatible algebras}

In this subsection we work out some details of the $\mathbb{Z}_{2} \times \mathbb{Z}_{2}$ orbifold since this case will be important in the next section. Rather than taking each group space $G$ and check whether its structure constants are compatible with the $\mathbb{Z}_{2} \times \mathbb{Z}_{2}$ orbifold action, we work with a generic group space with one-forms $e^{a}, a=1, \ldots, 6$ that satisfy $\mathrm{d} e^{a}=-\frac{1}{2} f_{b c}^{a} e^{b} \wedge e^{c}$. We check which structure constants $f_{b c}^{a}$, and therefore which groups, are compatible with the $\mathbb{Z}_{2} \times \mathbb{Z}_{2}$ orbifold action and a further orientifold action. For non-compact $G$ one also has to verify the existence and compatibility of a lattice $L$ such that $G / L$ is compact.

Defining the three complex one-forms

$$
\begin{aligned}
\mathrm{d} z^{1} & =e^{1}+\tau_{1} e^{6}, \\
\mathrm{~d} z^{2} & =e^{2}+\tau_{2} e^{4}, \\
\mathrm{~d} z^{3} & =e^{3}+\tau_{3} e^{5},
\end{aligned}
$$

where the $\tau_{i}$ are complex complex structure moduli, we find from (4.2a) that the $\mathbb{Z}_{2} \times \mathbb{Z}_{2}$

\footnotetext{
${ }^{15}$ These orbifold fixed points should not be confused with the fixed loci of the orientifold action. The cycles that are fixed under the elements $\gamma \sigma$ with $\gamma \in \Gamma$ are not singularities but rather the cycles wrapped by the orientifold sources.

${ }^{16}$ We refrain from using a more natural definition like $\mathrm{d} z^{i}=e^{i}+\tau_{i} e^{i+3}, i=1,2,3$, in order to match with [19] where the authors partially classified group spaces that are compatible with the $\mathbb{Z}_{2} \times \mathbb{Z}_{2}$ orbifolds.
} 
action is generated by $\theta_{1}$ and $\theta_{2}$ that act as

$$
\theta_{1}:\left\{\begin{array}{ll}
e^{1} & \rightarrow-e^{1} \\
e^{2} & \rightarrow-e^{2} \\
e^{3} & \rightarrow e^{3} \\
e^{4} & \rightarrow-e^{4} \\
e^{5} & \rightarrow e^{5} \\
e^{6} & \rightarrow-e^{6}
\end{array} \quad \theta_{2}:\left\{\begin{array}{l}
e^{1} \rightarrow-e^{1} \\
e^{2} \rightarrow e^{2} \\
e^{3} \rightarrow-e^{3} \\
e^{4} \rightarrow e^{4} \\
e^{5} \rightarrow-e^{5} \\
e^{6} \rightarrow-e^{6}
\end{array} .\right.\right.
$$

For this orbifold there are two possible orientifold projections [9]. Here we will focus on the so called standard orientifold projection $\sigma: z^{i} \rightarrow \bar{z}^{i}, i=1,2,3$. We will present the relevant results for a classification for the non-standard orientifold projection $\sigma_{n s}:\left(z^{1}, z^{2}, z^{3}\right) \rightarrow$ $\left(\bar{z}^{1}, \bar{z}^{3}, \bar{z}^{2}\right)$ in the next subsection.

An orientifold action always projects out half of the complex structure moduli. For the standard orientifold projection this simply results in $\operatorname{Re}\left(\tau_{i}\right)=0, \forall i$. The explicit action of $\sigma$ on the $e^{i}$ is given by

$$
\sigma:\left\{\begin{array}{l}
e^{1} \rightarrow e^{1} \\
e^{2} \rightarrow e^{2} \\
e^{3} \rightarrow e^{3} \\
e^{4} \rightarrow-e^{4} \\
e^{5} \rightarrow-e^{5} \\
e^{6} \rightarrow-e^{6}
\end{array}\right.
$$

This restricts the Lie algebra to be of the particular form

$$
\begin{array}{rlrl}
\mathrm{d} e^{1} & =f^{1}{ }_{23} e^{23}+f^{1}{ }_{45} e^{45}, & \mathrm{~d} e^{2}=f^{2}{ }_{13} e^{13}+f^{2}{ }_{56} e^{56}, \\
\mathrm{~d} e^{3}=f^{3}{ }_{12} e^{12}+f^{3}{ }_{46} e^{46}, & \mathrm{~d} e^{4}=f^{4}{ }_{36} e^{36}+f^{4}{ }_{15} e^{15}, \\
\mathrm{~d} e^{5}=f^{5}{ }_{14} e^{14}+f^{5}{ }_{26} e^{26}, & \mathrm{~d} e^{6}=f^{6}{ }_{34} e^{34}+f^{6}{ }_{25} e^{25} .
\end{array}
$$

We find that the algebra is automatically unipotent.

From the combination of the orientifold with the orbifold elements we find four intersecting O6 planes in the covering space $G$

\begin{tabular}{|c|c|c|c|c|c|}
\hline$e^{1}$ & $e^{2}$ & $e^{3}$ & $e^{4}$ & $e^{5}$ & $e^{6}$ \\
\hline \hline$\bigotimes$ & $\bigotimes$ & $\bigotimes$ & - & - & - \\
\hline- & - & $\bigotimes$ & $\bigotimes$ & - & $\bigotimes$ \\
\hline- & $\bigotimes$ & - & - & $\bigotimes$ & $\bigotimes$ \\
\hline$\bigotimes$ & - & - & $\bigotimes$ & $\bigotimes$ & - \\
\hline
\end{tabular}

where each entry denotes a left-invariant direction of $G$.

We want to determine all the unipotent algebras in our classification that are consistent with the $\mathbb{Z}_{2} \times \mathbb{Z}_{2}$ orbifold and the standard orientifold. Let us first do this on the level of the structure constants and later link it to the algebras in our classification tables. From 
now on we group the structure constants into a matrix denoted $r$ as in [9]

$$
r=\left(\begin{array}{cccc}
f^{1}{ }_{45} & f^{1}{ }_{23} & -f^{6}{ }_{34} & -f^{6}{ }_{25} \\
-f^{2}{ }_{56} & f^{4}{ }_{36} & -f^{2}{ }_{13} & -f^{4}{ }_{15} \\
-f^{3}{ }_{46} & f^{5}{ }_{26} & f^{5}{ }_{14} & f^{3}{ }_{12}
\end{array}\right) .
$$

To classify all possible Lie algebras that are invariant under $\theta_{1}, \theta_{2}$ and $\sigma$ we need to understand what kind of $\mathrm{GL}(6, \mathbb{R})$-transformations commute with $\theta_{1}, \theta_{2}$ and $\sigma$. Then we have to mod out by these transformations in order to classify inequivalent models. Hence, consider a general transformation 6 by 6 matrix $M \in \mathrm{GL}(6, \mathbb{R})$ that acts on the generators

$$
e^{i} \rightarrow e^{i}=M_{j}^{i} e^{j}
$$

The condition that $M$ commutes with $\theta_{1}, \theta_{2}$ and $\sigma$ and thus preserves the form of the Maurer-Cartan equations leads to

$$
M=\operatorname{diag}\left(\lambda_{1}, \lambda_{2}, \lambda_{3}, \lambda_{4}, \lambda_{5}, \lambda_{6}\right)
$$

where $\lambda_{i} \neq 0$ corresponds to a rescaling of a generator. Since there are six $\lambda$ 's to choose from one can set up to six non-zero $f_{b c}^{a}$ 's equal to \pm 1 . Then one can check that the Jacobi identities ensure that the other 6 remaining $f_{b c}^{a}$ 's have to be either \pm 1 or 0 . So we have found that the only $M$-transformations that preserve the above form of the Maurer-Cartan equations are rescalings and that these rescalings ensure that all structure constants can be normalized to $\pm 1,0$.

So now we can start investigating all sets of matrices $r$ with entries $0,+1,-1$ that obey the Jacobi identities. If we exclude the trivial algebra then there is at least one structure constant non-zero. After relabeling of the axes we can always take that to be the top-left element of $r, f^{1}{ }_{45} \neq 0$.

If we go through all Jacobi identities, we find four classes of allowed algebras:

$$
\begin{aligned}
& \text { class } A: \quad r=\left(\begin{array}{cccc}
f^{1}{ }_{45} & f^{1}{ }_{23} & -f^{6}{ }_{34} & -f^{6}{ }_{25} \\
-f^{2}{ }_{56} & \frac{f^{1}{ }_{23} f^{2}{ }^{2}}{f^{1}{ }^{45}} & -\frac{f^{2}{ }_{56} f^{6}{ }^{6}}{f^{1} 45} & \frac{f^{6}{ }_{25}^{2} f_{56}}{f^{1}{ }^{1}} \\
-f^{3}{ }_{46} & \frac{f^{1}{ }_{23} f^{3} 46}{f^{1} 45} & \frac{f^{6}{ }^{1} f^{3} 46}{f^{1} 45} & -\frac{f^{3} 46 f^{6}}{f^{1} 45}
\end{array}\right) \text {, } \\
& \text { class } B: \quad r=\left(\begin{array}{cccc}
f^{1}{ }_{45} & 0 & 0 & -f^{6}{ }^{25} \\
-f^{2}{ }_{56} & 0 & 0 & -f^{4}{ }_{15} \\
0 & 0 & 0 & 0
\end{array}\right) \text {, } \\
& \text { class } C: \quad r=\left(\begin{array}{cccc}
f^{1}{ }_{45} & f^{1}{ }_{23} & -f^{6}{ }_{34} & 0 \\
0 & 0 & 0 & 0 \\
-f^{3}{ }_{46} & \frac{f^{1}{ }_{23} f^{3} 46}{f^{1} 45} & f^{5}{ }_{14} & 0
\end{array}\right) \text {, } \\
& \text { class } D: \quad r=\left(\begin{array}{cccc}
f^{1} 45 & 0 & 0 & 0 \\
0 & 0 & 0 & -f^{4} 15 \\
0 & 0 & f^{5} 14 & 0
\end{array}\right) \text {. }
\end{aligned}
$$


We further discuss some of these four classes and their corresponding group spaces in section 5.1 .

Note that we obtained the four cases through relabeling of the generators, in other words by considering permutations. Permutations are not of the form $M$ as given in equation (4.10) since permutations change the definition of the various involutions. However, one can show for the standard orientifold, that permutations of rows and columns in $r$ map one model to an equivalent one.

\section{The SU(3)-structure}

There are no one-forms that are invariant under the $\mathbb{Z}_{2} \times \mathbb{Z}_{2}$ orbifold action. Furthermore, the only invariant two-forms have all negative parity under the orientifold action $\sigma$. We choose them to be

$$
\begin{aligned}
& Y_{1}^{(2-)}=\frac{i}{2 \hat{\tau}_{1}} \mathrm{~d} z^{1} \wedge \mathrm{d} \bar{z}^{1}=e^{16}, \\
& Y_{2}^{(2-)}=-\frac{i}{2 \hat{\tau}_{2}} \mathrm{~d} z^{2} \wedge \mathrm{d} \bar{z}^{2}=-e^{24}, \\
& Y_{3}^{(2-)}=\frac{i}{2 \hat{\tau}_{3}} \mathrm{~d} z^{3} \wedge \mathrm{d} \bar{z}^{3}=e^{35},
\end{aligned}
$$

where $\hat{\tau}_{i} \equiv \operatorname{Im}\left(\tau_{i}\right)$. The even and odd real three-forms are

$$
\begin{aligned}
Y_{1}^{(3+)} & =e^{123}, \quad Y_{2}^{(3+)}=e^{145}, \quad Y_{3}^{(3+)}=-e^{256}, \quad Y_{4}^{(3+)}=-e^{346}, \\
Y^{(3-) 1} & =e^{456}, \quad Y^{(3-) 2}=e^{236}, \quad Y^{(3-) 3}=e^{134}, \quad Y^{(3-) 4}=e^{125} .
\end{aligned}
$$

Since $J$ and $\Omega_{R}$ must be odd under the $\mathrm{SU}(3)$-structure preserving orientifold involution, we find that they must be of the form 17

$$
\begin{aligned}
& J=k^{i} Y_{i}^{(2-)}=k^{1} e^{16}-k^{2} e^{24}+k^{3} e^{35}, \\
& \Omega_{R}=\mathcal{F}_{K} Y^{(3-) K}=\mathcal{F}_{1} e^{456}+\mathcal{F}_{2} e^{236}+\mathcal{F}_{3} e^{134}+\mathcal{F}_{4} e^{125},
\end{aligned}
$$

where $k^{i}$ and $\mathcal{F}_{K}$ are real coefficients.

From

$$
J \wedge J \wedge J=6 k^{1} k^{2} k^{3} e^{123456},
$$

we find that (for our choice of orientation) $k^{1} k^{2} k^{3}>0$ rendering all or one of the coefficients $k^{i}$ positive. This allows us to compute the complex structure $I$. In order to be able to properly normalize it with real $c$ in $(\underline{B .2})$ we need furthermore $\mathcal{F}_{1} \mathcal{F}_{2} \mathcal{F}_{3} \mathcal{F}_{4}>0$. Explicitly we find

$$
\begin{array}{ll}
I_{6}^{1}=-\sqrt{\frac{\mathcal{F}_{1} \mathcal{F}_{2}}{\mathcal{F}_{3} \mathcal{F}_{4}}}, & I_{1}^{6}=\sqrt{\frac{\mathcal{F}_{3} \mathcal{F}_{4}}{\mathcal{F}_{1} \mathcal{F}_{2}}}, \\
I_{4}^{2}=+\sqrt{\frac{\mathcal{F}_{1} \mathcal{F}_{3}}{\mathcal{F}_{2} \mathcal{F}_{4}}}, & I_{2}^{4}=-\sqrt{\frac{\mathcal{F}_{2} \mathcal{F}_{4}}{\mathcal{F}_{1} \mathcal{F}_{3}}}, \\
I_{5}^{3}=-\sqrt{\frac{\mathcal{F}_{1} \mathcal{F}_{4}}{\mathcal{F}_{2} \mathcal{F}_{3}}}, & I_{3}^{5}=\sqrt{\frac{\mathcal{F}_{2} \mathcal{F}_{3}}{\mathcal{F}_{1} \mathcal{F}_{4}}}
\end{array}
$$

\footnotetext{
${ }^{17}$ Note, that we have relabeled the expansion coefficient and flipped the sign in front of $e^{24}$ compared to 19.
} 
From equation (B.3) we obtain the metric, which turns out to be diagonal, consistent with even parity under the orientifold involution

$$
g=\frac{1}{\sqrt{\mathcal{F}_{1} \mathcal{F}_{2} \mathcal{F}_{3} \mathcal{F}_{4}}}\left(k^{1} \mathcal{F}_{3} \mathcal{F}_{4}, k^{2} \mathcal{F}_{2} \mathcal{F}_{4}, k^{3} \mathcal{F}_{2} \mathcal{F}_{3}, k^{2} \mathcal{F}_{1} \mathcal{F}_{3}, k^{3} \mathcal{F}_{1} \mathcal{F}_{4}, k^{1} \mathcal{F}_{1} \mathcal{F}_{2}\right)
$$

With the metric available we can compute $\Omega_{I}=\star \Omega_{R}$

$$
\Omega_{I}=\mathcal{Z}^{K} Y_{K}^{(3+)}=\sqrt{\mathcal{F}_{1} \mathcal{F}_{2} \mathcal{F}_{3} \mathcal{F}_{4}}\left(\mathcal{F}_{1}^{-1} e^{123}+\mathcal{F}_{2}^{-1} e^{145}-\mathcal{F}_{3}^{-1} e^{256}-\mathcal{F}_{4}^{-1} e^{346}\right) .
$$

The normalization condition (B.1) leads to

$$
\sqrt{\mathcal{F}_{1} \mathcal{F}_{2} \mathcal{F}_{3} \mathcal{F}_{4}}=k^{1} k^{2} k^{3}
$$

The normalization condition (B.1) also allows us to determine the normalization of

$$
\Omega=\mathcal{F}_{K} Y^{(3-) K}+i \mathcal{Z}^{K} Y_{K}^{(3+)} \propto \mathrm{d} z^{1} \wedge \mathrm{d} z^{2} \wedge \mathrm{d} z^{3}
$$

so that we can express the $\mathcal{Z}^{K}$ and $\mathcal{F}_{K}$ explicitly in terms of the three independent complex structure parameters $\hat{\tau}_{i} \equiv \operatorname{Im}\left(\tau_{i}\right)$ and the $k^{i}$

$$
\begin{aligned}
& \mathcal{Z}^{1}=\frac{1}{\sqrt{\hat{\tau}_{1} \hat{\tau}_{2} \hat{\tau}_{3}}}, \quad \mathcal{Z}^{2}=-\sqrt{\frac{\hat{\tau}_{2} \hat{\tau}_{3}}{\hat{\tau}_{1}}}, \quad \mathcal{Z}^{3}=\sqrt{\frac{\hat{\tau}_{1} \hat{\tau}_{3}}{\hat{\tau}_{2}}}, \quad \mathcal{Z}^{4}=-\sqrt{\frac{\hat{\tau}_{1} \hat{\tau}_{2}}{\hat{\tau}_{3}}}, \\
& \mathcal{F}_{K}=\frac{\sqrt{k^{1} k^{2} k^{3}}}{\mathcal{Z}^{K}}, \quad \forall K .
\end{aligned}
$$

The absence of nowhere-vanishing one-forms on homogeneous strict SU(3)-structure spaces implies $W_{4}=W_{5}=0$ 18. Since there are no even two-forms in this example there are no gauge fields and no D-terms, so that we find that $W_{1}, W_{2}$ are real and we obtain a halfflat SU(3)-structure space (cf. appendix (B)). Furthermore, we can construct the remaining torsion classes from the identities 19

$$
\begin{aligned}
& W_{1}=-\frac{1}{6} \star_{6}\left(\mathrm{~d} J \wedge \Omega_{I}\right), \\
& W_{2}=-\star \mathrm{d} \Omega_{I}+2 W_{1} J, \\
& W_{3}=\mathrm{d} J-\frac{3}{2} W_{1} \Omega_{R} .
\end{aligned}
$$

\subsection{The non-standard $\mathbb{Z}_{2} \times \mathbb{Z}_{2}$ orientifold example}

We provide some details for an alternative orientifold projection of the $\mathbb{Z}_{2} \times \mathbb{Z}_{2}$ orbifold, which leads to explicit examples of $4 \mathrm{D}$ supergravity theories with D-terms.

\footnotetext{
${ }^{18}$ For non-homogeneous $\mathrm{SU}(3)$-structures this is not true as forms $W_{4}$ and $W_{5}$ that vanish somewhere are still allowed.

${ }^{19}$ To understand how the torsion classes depend on all moduli is not too hard for this simple example, but formulae for more general cases have been derived in [40].
} 
We take the $\mathbb{Z}_{2} \times \mathbb{Z}_{2}$ orbifold group to act on the six one-forms $e^{a}$ as

$$
\theta_{1}:\left\{\begin{array}{l}
e^{1} \rightarrow-e^{1} \\
e^{2} \rightarrow-e^{2} \\
e^{3} \rightarrow e^{3} \\
e^{4} \rightarrow-e^{4} \\
e^{5} \rightarrow-e^{5} \\
e^{6} \rightarrow e^{6}
\end{array} \quad \theta_{2}:\left\{\begin{array}{l}
e^{1} \rightarrow-e^{1} \\
e^{2} \rightarrow e^{2} \\
e^{3} \rightarrow-e^{3} \\
e^{4} \rightarrow-e^{4} \\
e^{5} \rightarrow e^{5} \\
e^{6} \rightarrow-e^{6}
\end{array}\right.\right.
$$

Due to the simplicity of this action we see that it does not relate any of the $f_{b c}^{a}$ to each other, rather it projects out all but 24 of the metric fluxes. In the case of the standard orientifold projection 12 more metric fluxes get projected out by the orientifold projection. However, for the non-standard orientifold projection $\sigma_{\text {ns }}$ we have the following action on the $e^{a}$

$$
\sigma_{\mathrm{ns}}:\left\{\begin{aligned}
e^{1} & \rightarrow e^{1} \\
e^{2} & \rightarrow e^{3} \\
e^{3} & \rightarrow e^{2} \\
e^{4} & \rightarrow-e^{4} \\
e^{5} & \rightarrow-e^{6} \\
e^{6} & \rightarrow-e^{5}
\end{aligned}\right.
$$

$\sigma_{\mathrm{ns}}$ relates 10 of the metric fluxes to each other

$$
\begin{array}{ll}
f^{1}{ }_{26}=-f^{1}{ }_{35}, & f^{4}{ }_{35}=f^{4}{ }_{26}, \\
f^{2}{ }_{13}=f^{3}{ }_{12}, & f^{5}{ }_{13}=-f^{6}{ }_{12}, \\
f^{2}{ }_{16}=-f^{3}{ }_{15}, & f^{5}{ }_{16}=f^{6}{ }_{15}, \\
f^{2}{ }_{34}=-f^{3}{ }_{24}, & f^{5}{ }_{34}=f^{6}{ }_{24}, \\
f^{2}{ }_{46}=f^{3}{ }_{45}, & f^{5}{ }_{46}=-f^{6}{ }_{45},
\end{array}
$$

and projects out two: $f^{1}{ }_{23}=f^{1}{ }_{56}=0$.

It is straightforward to check that we have two odd and one even two-form which we take to be

$$
Y_{1}^{(2-)}=-\frac{1}{2} e^{14}, \quad Y_{2}^{(2-)}=e^{25}+e^{36}, \quad Y^{(2+)}=e^{25}-e^{36} .
$$

So in this case we have two matrices $r_{i K}$ and $\hat{r}_{K}$ such that

$$
\mathrm{d} Y_{i}^{(2-)}=-r_{i K} Y^{(3-) K}, i=1,2, \quad \mathrm{~d} Y^{(2+)}=-\hat{r}^{K} Y_{K}^{(3+)}, K=1,2,3,4 .
$$

Choosing the three-forms to be

$$
\begin{array}{ll}
Y_{1}^{(3+)}=e^{126}-e^{135}, & Y^{(3-) 1}=\frac{1}{2}\left(e^{246}-e^{345}\right), \\
Y_{2}^{(3+)}=-e^{234}, & Y^{(3-) 2}=e^{156} \\
Y_{3}^{(3+)}=-e^{456}, & Y^{(3-) 3}=e^{123} \\
Y_{4}^{(3+)}=-e^{246}-e^{345}, & Y^{(3-) 4}=-\frac{1}{2}\left(e^{126}+e^{135}\right),
\end{array}
$$


we find the explicit expressions

$$
\begin{aligned}
r & =\left(\begin{array}{cccc}
f_{26}^{1} & \frac{1}{2} f_{56}^{4} & \frac{1}{2} f_{23}^{4} & -f_{26}^{4} \\
2\left(f_{24}^{3}+f_{45}^{6}\right) & 2 f_{15}^{3} & -2 f_{12}^{6} & -2\left(f_{12}^{3}+f_{15}^{6}\right)
\end{array}\right), \\
\hat{r} & =\left(\begin{array}{llll}
-f_{12}^{3}+f_{15}^{6} & 2 f_{24}^{6} & 2 f_{45}^{3} & f_{24}^{3}-f_{45}^{6}
\end{array}\right) .
\end{aligned}
$$

The matrices are again constrained by the requirement that $\mathrm{d}^{2} e^{a} \propto f_{b[c}^{a} f_{d e]}^{b}=0, \forall a$. This gives in our case 11 independent constraints; only two of these are obtained by demanding that $\mathrm{d}^{2} Y_{i}^{(2-)}=r_{i K} \hat{r}^{K} Y^{(4+)}=0, i=1,2$. Furthermore, we have to satisfy $\mathrm{d} H=h_{K} \mathrm{~d} Y^{(3-) K}=h_{K} \hat{r}^{K} Y^{(4+)}=0$, i.e. the metric fluxes have to satisfy $h_{K} \hat{r}^{K}=0$.

\subsection{General abelian and non-abelian orbifolds}

Contrary to for example the torus $\mathrm{U}(1)^{6}=T^{6}=\mathbb{R}^{6} / L$, for any compact group space it is not necessary to mod out by a lattice $L$ so that for these group spaces the only constraint on the orbifold group is that it is compatible with the structure constants. This means that in principle all the discrete subgroups of $\mathrm{SU}(3)$ should be studied in the search for dS vacua. In [9] the authors investigated the existence of dS extrema in 17 abelian orbifolds of small order and found that all of their examples except $\mathbb{Z}_{2} \times \mathbb{Z}_{2}$ can be excluded by no-go theorems. Intuitively the reason for this seems to be that quotients by orbifold groups of large order lead to models with only very few moduli and structure constant. $\mathbb{Z}_{2} \times \mathbb{Z}_{2}$ is the most symmetric case which allows for the largest number of moduli and structure constants and might therefore be the only abelian orbifold that leads to dS vacua. However, there is no proof of this and it is also possible that an abelian orbifold group that has not been studied yet allows for dS vacua. We do not pursue this here but leave it to the interested reader to construct such models or find no-go theorems.

Non-abelian orbifolds have not been studied at all in the context of de Sitter model building 20 Here, we would like to point out that they can be excluded if they contain an abelian subgroup that has already been excluded. So for example: based on the results of [9], we find that $\Delta\left(3 n^{2}\right)$ and $\Delta\left(6 n^{2}\right)$ are excluded for $n=3,4,6$. We will discuss the interesting case $n=2$ below. Since there are only finitely many forms and structure constants on a six-dimensional group space, one expects, as we argued above, that discrete subgroups of small order are the most promising because they lead to the richest set of fields and structure constants. Based on this observation it seems unlikely that $\Delta\left(3 n^{2}\right)$ and $\Delta\left(6 n^{2}\right)$ for $n=5,7,8, \ldots$ or any of the exceptional non-abelian groups (which are of order 36 or larger) can lead to dS vacua. Therefore we will focus on $\Delta(12)$ which is important for our construction of $\mathrm{dS}$ vacua in the next section.

\footnotetext{
${ }^{20}$ The so called isotropic $\mathbb{Z}_{2} \times \mathbb{Z}_{2}$ orbifold that was studied in [14,15] is the non-abelian orbifold $\Delta(12)$.
} 


\subsubsection{The standard orientifold of $\Delta(12)$}

For the standard orientifold projection, we find that the $\mathbb{Z}_{3}$ extension of $\mathbb{Z}_{2} \times \mathbb{Z}_{2}$ acts on the $e^{i}$ a: 21

$$
\mathbb{Z}_{3}:\left\{\begin{array}{ll}
e^{1} & \rightarrow e^{2} \\
e^{2} & \rightarrow e^{3} \\
e^{3} & \rightarrow e^{1} \\
e^{4} & \rightarrow-e^{5} \\
e^{5} & \rightarrow e^{6} \\
e^{6} & \rightarrow-e^{4}
\end{array} .\right.
$$

This leads to an identification of the moduli in (4.15) so that there is only one Kähler modulus $k \equiv k^{1}=k^{2}=k^{3}$ and two complex structure parameter $\mathcal{F}_{1}$ and $\mathcal{F}_{2}=\mathcal{F}_{3}=\mathcal{F}_{4}$. Furthermore, the structure constants have to satisfy the following additional constraints

$$
\begin{aligned}
& f^{1}{ }_{45}=-f^{2}{ }_{56}=-f^{3}{ }_{46}, \\
& f^{1}{ }_{23}=-f^{2}{ }_{13}=f^{3}{ }_{12}, \\
& f^{6}{ }_{34}=f^{4}{ }_{15}=-f^{5}{ }_{26} \\
& f^{6}{ }_{25}=-f^{4}{ }_{36}=-f^{5}{ }_{14} .
\end{aligned}
$$

The Jacobi identities can only be solved if $f^{1}{ }_{23}=f^{6}{ }_{34}=f^{6}{ }_{25}$ so that we find the $r$-matrix

$$
r=\left(\begin{array}{ll}
f^{1}{ }_{45} & -f^{1}{ }_{23}
\end{array}\right) .
$$

The structure constants can again be rescaled so that they are $0, \pm 1$.

\subsubsection{The non-standard orientifold of $\Delta(12)$}

For the non-standard orientifold projection (see 4.3 for details) the extension $\mathbb{Z}_{3}$ acts as

$$
\mathbb{Z}_{3}:\left\{\begin{array}{l}
e^{1} \rightarrow e^{2} \\
e^{2} \rightarrow e^{3} \\
e^{3} \rightarrow e^{1} \\
e^{4} \rightarrow e^{5} \\
e^{5} \rightarrow e^{6} \\
e^{6} \rightarrow e^{4}
\end{array}\right.
$$

This quotient projects out the even two-form so that there is no gauge field and therefore no D-term anymore. We are again left with a model that has one Kähler modulus and two complex structure parameters. The extra $\mathbb{Z}_{3}$ quotient requires that the structure constants

\footnotetext{
${ }^{21}$ The two extra minus signs arise due to the minus sign in our definition of $Y_{2}^{(2-)}$ in (4.12).
} 
satisfy the constraints

$$
\begin{aligned}
f^{1}{ }_{26} & =f^{3}{ }_{15}=-f^{3}{ }_{24}, \\
f^{4}{ }_{26} & =f^{6}{ }_{15}=f^{6}{ }_{24}, \\
f^{4}{ }_{23} & =f^{6}{ }_{12}, \\
f^{4}{ }_{56} & =f^{6}{ }_{45}, \\
f^{3}{ }_{12} & =f^{3}{ }_{45}=0 .
\end{aligned}
$$

This case is peculiar since there are four independent structure constants but only two linear combinations appear in the $r$-matrix

$$
r=\left(\begin{array}{ll}
2 f^{1}{ }_{26}-f_{56}^{4} & f^{4}{ }_{23}
\end{array}\right) .
$$

The existence of extra structure constants that do not appear in the $r$ - or $\hat{r}$-matrices, and therefore not in the scalar potential, was previously noticed for some abelian-orbifolds in [9. These extra structure constants appear in the Jacobi identities. For $r \neq 0$ the Jacobi identities can only be solved for

$$
\begin{aligned}
f_{26}^{4} & =0, \quad f_{26}^{1}=0, \quad f_{23}^{4}=0, \\
\text { or } \quad f^{4}{ }_{26} & =0, \quad f^{1}{ }_{26}=f^{4}{ }_{56} .
\end{aligned}
$$

\section{Classification of de Sitter solutions}

As we have argued at the end of section 2.1. flux compactifications of massive type IIA together with O6-planes lead to a 4D scalar potential that depends on all moduli. Among the moduli there are two universal ones that are present for any compactification space, the volume modulus $\rho=\left(\operatorname{vol}_{6}\right)^{1 / 3}$ and the dilaton $\phi$. Based on the scaling behavior of the scalar potential with respect to these two fields, the authors of [4] showed that type IIA flux compactification with RR- and $H$-flux and O6-planes cannot lead to dS vacua when the compact space is a $C Y_{3}$ manifold. However, this no-go theorem can be circumvented on $\mathrm{SU}(3)$-structure spaces with negative scalar curvature. This observation led to many papers [5, 7, 9-12] that generalized the no-go theorem of [4] but also to constructions of actual classical dS solutions from the 4D effective theory [5, 7, 9, 11, 13, 17] (see also [18]) as well as 10D constructions [10,19]. However, so far all explicit constructions of geometric dS solutions are perturbatively unstable since there is at least one tachyonic field. In compactifications on more general non-geometric spaces it is possible to find stable dS vacua [14, 15. However, since the internal space is non-geometric it is not clear whether the supergravity approach is reliable.

In [9] the authors studied twisted versions of all abelian toroidal orbifolds $T^{6} / \mathbb{Z}_{N}$ and $T^{6} / \mathbb{Z}_{N} \times \mathbb{Z}_{M}$ and checked whether they allow for dS solutions or can be excluded by nogo theorems. Since the orbifold and orientifold actions have to be compatible with the torus lattice, it turns out that there are 36 different cases [9, 69, 70]. However, if one 
restricts to the untwisted sector 22 only 11 of these models are different. Out of these 11 models only the two different orientifolds of the $\mathbb{Z}_{2} \times \mathbb{Z}_{2}$ orbifold could not be excluded by no-go theorems and were shown to allow for dS solutions. Only for the non-standard orientifold projection were the authors able to construct an explicit compact example. For the standard orientifold projection it was shown in [8] that the group space $\mathrm{SU}(2) \times \mathrm{SU}(2)$ provides an explicit compact example that allows for $\mathrm{dS}$ solutions. In this section we will fully classify all possible group spaces that lead to dS solutions and that are compatible with the $\mathbb{Z}_{2} \times \mathbb{Z}_{2}$ orbifold action and either one of the orientifold projections. As in the previous papers we establish the solutions numerically by minimizing the slow roll parameter

$$
\epsilon=G^{I J} \frac{\partial_{I} V \partial_{J} V}{2 V^{2}}
$$

where $G^{I J}$ denotes the inverse field space metric.

\subsection{The standard $\mathbb{Z}_{2} \times \mathbb{Z}_{2}$ orientifold}

In the previous section in equation (4.11) we have classified the group manifolds that are compatible with the $\mathbb{Z}_{2} \times \mathbb{Z}_{2}$ orbifold and the standard orientifold projection. In this section we check which of these group spaces can lead to dS vacua 23 .

Class $\mathrm{B}$ and $\mathrm{C}$ are excluded by a no-go theorem 9 since they have at least one zero row. Class $\mathrm{D}$ is of the form $G_{6}=G_{3} \times U(1)^{3}$, with $G_{3}$ a unipotent three-dimensional algebra. For this case there is no analytic no-go theorem, but numerically one finds $\epsilon \approx 1.57221$ unless $f^{1}{ }_{45}=f^{4}{ }_{15}=f^{5}{ }_{14}$ in which case one gets $\epsilon \approx 2$ (cf. [9]). For class A we make further subdivisions: The no-go theorem implies that $f^{2}{ }_{56}$ and $f^{3}{ }_{46}$ should be non-zero, otherwise the second, resp. the third row would vanish. Hence we have the following rule:

$$
f^{2}{ }_{56}, f^{3}{ }_{46} \in\{-1,+1\}, \quad f^{1}{ }_{23}, f^{6}{ }_{34}, f^{6}{ }_{25} \in\{-1,0,+1\} .
$$

When any of the $\left(f^{1}{ }_{23}, f^{6}{ }_{34}, f^{6}{ }_{25}\right)$ is zero, then the whole column is zero. Hence we consider the following subclasses

- class AI : all entries non-zero.

- class AII : one column zero.

- class AIII: two columns zero.

- class AIV : three columns zero.

\footnotetext{
${ }^{22}$ It would be very interesting to study the inclusion of the twisted sector. This has not been done in the literature and we refrain from doing it since the number of moduli would increase substantially, thus making the analysis of the scalar potential very complicated.

${ }^{23}$ The Mathematica package STRINGVACUA [71] is very useful in calculating the scalar potential for these models.
} 
For class AIV there is no no-go theorem but numerically one finds $\epsilon \approx \frac{4}{3}$ (cf. [9]).

Class AI comprise the three simple algebras: $\mathfrak{s o}(4), \mathfrak{s o}(2,2)$ and $\mathfrak{s o}(3,1)$, as can be easily verified by computing the Cartan-Killing metric. For these three algebras we can numerically get $\epsilon$ to vanish. Explicitly we have

$$
\begin{aligned}
r_{\mathfrak{s o}(4)} & =\left(\begin{array}{llll}
+1 & +1 & +1 & -1 \\
+1 & -1 & -1 & -1 \\
+1 & -1 & +1 & +1
\end{array}\right), \\
r_{\mathfrak{s o}(3,1)} & =\left(\begin{array}{llll}
-1 & +1 & +1 & -1 \\
-1 & -1 & -1 & -1 \\
-1 & -1 & +1 & +1
\end{array}\right),
\end{aligned}
$$

up to re-scalings of generators with \pm 1 .

Class AII consists out of algebras that are all not solvable, neither semi-simple, they are in between. It is sufficient to take the last column zero and the first three-non-zero. For instance, assume it is the third column instead that is zero. Then, by the permutation $\left(e_{3}, e_{4}\right) \leftrightarrow\left(e_{2}, e_{5}\right)$ we can push all zeros to the fourth column. Furthermore, by fixing the scaling of the generators $e_{1}, e_{2}, e_{3}$ we can always take the first column to consist of +1 's. A remaining scaling freedom is a simultaneous rescaling of $e_{4}, e_{5}, e_{6}$ with \pm 1 . So we find the algebras

$$
\begin{aligned}
r_{a}=\left(\begin{array}{llll}
+1 & +1 & +1 & 0 \\
+1 & -1 & -1 & 0 \\
+1 & -1 & +1 & 0
\end{array}\right), & r_{b}=\left(\begin{array}{llll}
+1 & +1 & -1 & 0 \\
+1 & -1 & +1 & 0 \\
+1 & -1 & -1 & 0
\end{array}\right), \\
r_{c}=\left(\begin{array}{llll}
+1 & -1 & +1 & 0 \\
+1 & +1 & -1 & 0 \\
+1 & +1 & +1 & 0
\end{array}\right), & r_{d}=\left(\begin{array}{llll}
+1 & -1 & -1 & 0 \\
+1 & +1 & +1 & 0 \\
+1 & +1 & -1 & 0
\end{array}\right) .
\end{aligned}
$$

All of these give vanishing $\epsilon$. These algebras must be contained in table 2. One can verify that generators $T_{3}, T_{4}, T_{6}$ form the semi-simple piece and that the generators $T_{1}, T_{2}, T_{5}$ form the radical. It is then straightforward to read off the representation $\rho$. We find that

$$
\begin{aligned}
& \mathfrak{s o}(3) \ltimes_{3} \mathfrak{u}(1)^{3} \quad \text { is given by } r_{a}, \\
& \mathfrak{s o}(2,1) \ltimes_{3} \mathfrak{u}(1)^{3} \quad \text { is given by } \quad r_{b}, r_{c}, r_{d} .
\end{aligned}
$$

Thus, only two out of these four sets of structure constants are independent.

Class AIII are all solvable algebras. We can take, without loss of generality, the last two columns to be zero and take the first column to be +1 . Then, we find the following two algebras

$$
r_{\text {solvable } 1}=\left(\begin{array}{cccc}
+1 & +1 & 0 & 0 \\
+1 & -1 & 0 & 0 \\
+1 & -1 & 0 & 0
\end{array}\right), \quad r_{\text {solvable } 2}=\left(\begin{array}{cccc}
+1 & -1 & 0 & 0 \\
+1 & +1 & 0 & 0 \\
+1 & +1 & 0 & 0
\end{array}\right)
$$

Both of these give vanishing $\epsilon$. Solvable2 equals the algebra s1.2 as can be seen by rescaling $e_{1}$ and $e_{2}$ by -1 to obtain the expression of [19]. This case is the dS example with the 
standard orientifold of [9]. The second solvable algebra does not appear in the table of [56] and implies that it is not algebraic. But, as indicated in [56] also s1.2 is not known to be algebraic. As a consequence we do not know whether these solvmanifolds can be compactified by dividing by an appropriate lattice $L$.

\subsection{The non-standard $\mathbb{Z}_{2} \times \mathbb{Z}_{2}$ orientifold}

This case is substantially less symmetric than the standard orientifold projection so that we refrain from explicitly spelling out every single solution to the Jacobi identities in terms of the $r$ and $\hat{r}$ matrices. However, it is straightforward and analogous to the previous subsection to work out all the details. Two of the Jacobi identities are

$$
r_{11} \hat{r}^{1}=0, \quad r_{11} r_{21}=0
$$

This suggest the classification:

- $A_{n s}: r_{11} \neq 0, r_{21}=0, \hat{r}^{1}=0$

- $B_{n s}: r_{11}=0, r_{21}=0, \hat{r}^{1} \neq 0$

- $C_{n s}: r_{11}=0, r_{21} \neq 0, \hat{r}^{1}=0$

- $D_{n s}: r_{11}=0, r_{21} \neq 0, \hat{r}^{1} \neq 0$

- $E_{n s}: r_{11}=0, r_{21}=0, \hat{r}^{1}=0$

For each of these classes we have to satisfy an additional 9 Jacobi identities and the constraint that $h_{K} \hat{r}^{K}=0$. It is straightforward to work out the generic solution for each class. Since we are interested in the existence of dS vacua we recall the no-go theorems derived in [9] which tell us that dS extrema and slow-roll inflation are excluded if one of these three conditions is satisfied

- $r_{1 K}=0, \forall K$

- $r_{2 K}=\hat{r}^{K}=0, \forall K$,

- $r_{a 1}=h_{1}=\hat{r}^{L}=0$ for $a=1,2, L=2,3,4$.

For the class $B_{n s}$ the 9 additional Jacobi identities require $\hat{r}^{2}=\hat{r}^{3}=\hat{r}^{4}=0$ and $h_{1}=0$ so that this entire class is excluded. In all of the other classes there are always solutions that fall under one of the above no-go theorems so that these cases do not lead to dS vacua. However, for classes $A_{n s}, D_{n s}, E_{n s}$ we were able to find dozens of numerical dS solutions that correspond to a variety of group spaces. 
In order to recognize equivalent models, one should divide out by those transformations that commute with the orbifold and orientifold involutions. Explicitly these transformations are given by a six-parameter family of matrices ( such that $\operatorname{det} M \neq 0$ )

$$
M=\left(\begin{array}{cccccc}
\lambda_{1} & 0 & 0 & 0 & 0 & 0 \\
0 & \lambda_{2} & 0 & 0 & \lambda_{3} & 0 \\
0 & 0 & \lambda_{2} & 0 & 0 & -\lambda_{3} \\
0 & 0 & 0 & \lambda_{4} & 0 & 0 \\
0 & \lambda_{5} & 0 & 0 & \lambda_{6} & 0 \\
0 & 0 & -\lambda_{5} & 0 & 0 & \lambda_{6}
\end{array}\right)
$$

In the standard case there were also transformations that did not commute with the involutions but that still mapped equivalent models to each other. This does not happen for the non-standard orientifold projection and complicates the analysis.

We find that the semi-simple algebras $\mathfrak{s o}(4), \mathfrak{s o}(3,1)$ and $\mathfrak{s o}(2,2)$ again allow for numerical de Sitter solutions. Of the non-solvable, non semi-simple algebras we found de Sitter solutions on $\mathfrak{s o}(2,1) \times \mathfrak{i s o}(1,1)$. Apart from that we have numerically found several examples of solvable algebras that allow de Sitter solutions, all are solvable of order 3 except one which is solvable of order 2. A typical example that is solvable of order 3 is

$$
\begin{aligned}
& f_{26}^{1}=1, \quad f_{35}^{1}=-1, \quad f_{46}^{2}=-\frac{1}{2}, \\
& f_{45}^{3}=-\frac{1}{2}, \quad f_{34}^{5}=\frac{1}{2}, \quad f_{24}^{6}=\frac{1}{2} .
\end{aligned}
$$

The only example that is solvable of order 2 reads

$$
\begin{aligned}
& f_{26}^{1}=1, \quad f_{35}^{1}=-1, \quad f_{16}^{2}=-\frac{1}{2}, \quad f_{46}^{2}=1 \\
& f_{15}^{3}=\frac{1}{2}, \quad f_{45}^{3}=1, \quad f_{26}^{4}=-1, \quad f_{35}^{4}=-1
\end{aligned}
$$

This example was already presented in [9]. Since it was constructed using the so-called base-fiber method it is manifestly compact.

Again, none of the nilpotent algebras allow de Sitter solutions. This was the same for the standard orientifold projection and one could wonder whether there is some no-go theorem that forbids de Sitter solutions for these spaces. The fact that nilmanifolds seem excluded is rather surprising since these manifolds are negatively curved for all values of the moduli, as opposed to the solvable cases or the semi-simple cases.

\subsection{The non-abelian orbifold $\Delta(12)$}

In order to construct dS solutions that are as simple as possible, one wants to investigate the possibility of taking a further quotient of the above models. This potentially allows for dS solutions with a smaller number of fields that are easier to analyze. As we discussed above $\Delta\left(3 n^{2}\right)$ and $\Delta\left(6 n^{2}\right)$ for $n=2$ are extensions of the abelian group $\mathbb{Z}_{2} \times \mathbb{Z}_{2}$. It is straightforward to check that there are no three-forms that are compatible with $\Delta(24)$ and either of our two different orientifolds so that we will focus on $\Delta(12)$. 


\section{The standard $\Delta(12)$ orientifold}

There are four different cases (see subsection 4.4.1 for details): For $f^{1}{ }_{45}=f^{1}{ }_{23}= \pm 1$ the algebra is $\mathfrak{s o}(4)$, for $f^{1}{ }_{45}=-f^{1}{ }_{23}= \pm 1$ the algebra is $\mathfrak{s o}(3,1)$, for $f^{1}{ }_{45}=0, f^{1}{ }_{23} \neq$ 0 we have $\mathfrak{s o}(3) \ltimes_{3} \mathfrak{u}(1)^{3}$ and finally for $f^{1} 45 \neq 0, f^{1}{ }_{23}=0$ we have case AIV from above. Numerically we find $\epsilon \geq \frac{27}{13}$ for $\mathfrak{s o}(3) \ltimes_{3} \mathfrak{u}(1)^{3}$ and $\epsilon \geq \frac{4}{3}$ for $\mathfrak{s o}(3,1)$ as well as the case AIV but we are able to find numerical dS solutions for $\mathfrak{s o}(4)$. The latter could have been anticipated since the dS solutions of [8, 14, 15, 19] are found by setting all Kähler and three complex structure moduli equal so that they are not only dS solutions of $\mathrm{SU}(2) \times \mathrm{SU}(2) / \mathbb{Z}_{2} \times \mathbb{Z}_{2}$ but also of $\mathrm{SU}(2) \times \mathrm{SU}(2) / \Delta(12)$.

\section{The non-standard $\Delta(12)$ orientifold}

In this case the existence of dS extrema depends only on the values of $2 f^{1}{ }_{26}-f^{4}{ }_{56}$ and $f^{4}{ }_{23}$ and not on $2 f^{1}{ }_{26}+f^{4}{ }_{56}$ and $f^{4}{ }_{26}$ as discussed in subsection 4.4.2. Numerically (up to rescalings) we only find $\mathrm{dS}$ extrema for $2 f^{1}{ }_{26}-f^{4}{ }_{56}=f^{4}{ }_{23}= \pm 1$. This was expected since the scalar potential for the standard and non-standard $\Delta(12)$ orientifold are identical and in the standard case there was only one choice for the $r$-matrix that allowed for $\mathrm{dS}$ solutions. The requirement that $f_{23}^{4} \neq 0$ together with the Jacobi identities (cf. 4.4.2) fully determines the algebra to be $\mathfrak{s o}(2,2)=\mathfrak{s o}(2,1) \times \mathfrak{s o}(2,1)$.

\subsection{The mass spectrum}

The explicit models we have discussed have up to 14 real moduli. In order for our dS critical points to be stable we have to demand that the masses of all these moduli are positive 24 . Since there is no supersymmetry that could ensure stability, the naïve expectation is that roughly half of the fields are tachyonic for a generic critical point. However, this is not the case. It turns out that all numerical solutions we found have always one tachyonic direction that is a mixture of all the moduli. This tachyonic direction is steep and leads to $\eta \sim O(1)$, so that these dS critical points are neither stable nor suitable for slow-roll inflation. For a large range of parameters there is only this one tachyon and the other up to 13 directions are stable. There are however also solutions where more directions are tachyonic $[19] 25$. Due to the complexity of the potential we were not able to find an analytic expression for the one seemingly universal tachyonic direction. In the simplest model the tachyonic direction seems to be determined by the root of an irreducible polynomial of degree 19 so that there is no hope of getting an analytic expression 26. It is not possible to tell whether this tachyon is generic for geometric SU(3)-structure flux compactification with O6-planes or whether it is model dependent. We would like to point out that the existence of the tachyon is independent of the orientifold projection and therefore the tachyon is present in

\footnotetext{
${ }^{24}$ Since we restrict to left-invariant forms, it is possible that there are unstable modes that are not captured by our consistent truncation.

${ }^{25}$ The models that are further truncations to just three complex moduli have always only one tachyonic direction.

${ }^{26}$ We thank Mike Stillman for studying the problem using the program Macaulay 2.
} 
two very different classes of models. Nevertheless, the orbifold group in the models that have dS critical points always contains $\mathbb{Z}_{2} \times \mathbb{Z}_{2}$ so that the tachyon might very well be related to this fact.

In a non-geometric compactification of an $\mathbb{Z}_{2} \times \mathbb{Z}_{2}$ orbifold there are new terms in the superpotential and those can lift the tachyon and lead to stable dS vacua [15]. It is not known whether it is possible to lift the tachyon in a geometric setup by adding further ingredients like $\mathrm{D} p$-branes, NS5-branes or KK-monopoles and it would be interesting to investigate these possibilities.

\section{Flux and charge quantization}

In addition to solving the supergravity equations of motion we have to take into account flux and charge quantization which are 'stringy constraints'. Whether a solution still exists after imposing these quantization conditions depends on how sensitive a solution is to a variation of the flux parameters. For instance, the SUSY AdS solutions of [6, 37, 52, 55] are quite robust against these variations since the flux parameters are not entirely fixed in the solutions and there are still many fluxes that can be chosen freely. This is in contrast with the de Sitter solutions, for which it has been observed that the solutions correspond to small "islands" in parameter space [10,19].

We will study the charge and flux quantization for the explicit example of a dS extremum found from a 4D approach in [8] and lifted to ten dimensions in [19]. The compact space is the standard orientifold projection of $\mathrm{SU}(2) \times \mathrm{SU}(2) / \mathbb{Z}_{2} \times \mathbb{Z}_{2}$ (see subsection 4.2). The dS extremum is also a dS extremum of $\mathrm{SU}(2) \times \mathrm{SU}(2) / \Delta(12)$ which means that all three Kähler moduli as well as three complex structure moduli are equal: $k \equiv k_{1}=k_{2}=k_{3}$ and $\mathcal{Z}_{2}=\mathcal{Z}_{3}=\mathcal{Z}_{4}$ (see subsection 4.4.1).

\subsection{Quantization of the dS solution on $\mathrm{SU}(2) \times \mathrm{SU}(2)$}

In appendix D we explain how we should in general quantize fluxes and charges in the presence of a non-trivial $H$-field. Let us now apply this to the dS solution of [19].

The bottom line of appendix $\mathrm{D}$ is that we should impose the quantization of $H$. As for the RR-fluxes, since the even $H$-twisted homology is

$$
H_{\text {even }}^{H}(\mathrm{SU}(2) \times \mathrm{SU}(2), \mathbb{Z})=\mathbb{Z}_{n_{H}},
$$

where the $\mathbb{Z}_{n_{H}}$ is spanned by the ordinary homology class of a point $[\{p\}]$, we need to impose quantization of the Romans mass $\hat{F}_{0}$. As we can infer from eq. (D.22) this implies the quantization of the charge of the source $j$. In fact, the orientifold charge for an O6plane should be -2 . However we can add D-branes on top of the O6-plane. Taking into account that the Maldacena-Núñez no-go theorem [72] requires the charge to be negative, it can thus be $-2,-1$ leading to

$$
\left(n_{H}, n_{\hat{F}_{0}}\right)=( \pm 1, \pm 2),( \pm 2, \pm 1),( \pm 1, \pm 1) .
$$


From the quantization of $\hat{F}_{0}$,

$$
\left(2 \pi l_{s}\right) \hat{F}_{0}=n_{\hat{F}_{0}},
$$

we find using (2.14) that

$$
f_{1}=\frac{e^{\Phi}}{2 \pi l_{s}} n_{\hat{F}_{0}}
$$

or factoring out the Kähler modulus $k, f_{i}=\hat{f}_{i} k^{-1 / 2}$,

$$
\hat{f}_{1}=e^{\Phi} \frac{k^{1 / 2}}{2 \pi l_{s}} n_{\hat{F}_{0}} .
$$

At this point it seems that we can have an internal space with large radius $\frac{k^{1 / 2}}{2 \pi l_{s}}$ and a small dilaton $e^{\Phi}$. From the quantization of $H$, eq. (D.13), we find

$$
C_{S^{3}} \frac{k}{\left(2 \pi l_{s}\right)^{2}} \hat{h}=n_{H}
$$

and for dS solution we have [19]

$$
\hat{h}=\frac{2\left(k^{6}-3 \mathcal{F}_{2}^{4}\right) \hat{f}_{5}+\sqrt{3}\left(k^{6}+\mathcal{F}_{2}^{4}\right) \hat{f}_{6}}{2 \mathcal{F}_{2}^{3} k^{3 / 2}}=\left(\mathcal{Z}_{2}^{3}-\frac{3}{\mathcal{Z}_{2}}\right) \hat{f}_{5}+\frac{\sqrt{3}}{2}\left(\mathcal{Z}_{2}^{3}+\frac{1}{\mathcal{Z}_{2}}\right) \hat{f}_{6},
$$

since $\mathcal{F}_{2}=\mathcal{F}_{3}=\mathcal{F}_{4}=\frac{k^{3 / 2}}{\mathcal{Z}_{2}}$ as explained in subsection 4.2.

For the supergravity regime we want to take the scale $k /\left(2 \pi l_{s}\right)^{2}$ large, so that the scale independent $\hat{h}$ should be small. However, this is impossible for the range of parameters that give $\mathrm{dS}$ solutions. To be more precise, in figure 2 we plot the quantities

$$
\hat{h}^{-1 / 2}=\left(C_{S^{3}} / n_{H}\right)^{1 / 2} \frac{k^{1 / 2}}{2 \pi l_{s}}, \quad \hat{f}_{1} \hat{h}^{1 / 2}=n_{\hat{F}_{0}}\left(n_{H} / C_{S^{3}}\right)^{1 / 2} e^{\Phi},
$$

for the entire range of parameters that give dS solutions. We see the volume is always small and the string coupling is always large. Numerically, at $f_{2} / f_{1}=0.9648$ (the edge of the family of dS solutions) $\hat{h}^{-1 / 2} \approx 0.4795$ and $\hat{f}_{1} \hat{h}^{1 / 2} \approx 4.554$, which are respectively the maximum and minimum. We conclude that flux quantization is impossible at tree-level in the supergravity regime i.e. there are no properly quantized solutions with large internal volume and small string coupling.

\section{Discussion}

In this paper we have reviewed the current status of constructing classical dS vacua in type IIA flux compactifications on SU(3)-structure manifolds with O6-planes. As summarized below, we presented also several new results and pointed out important future research directions. 


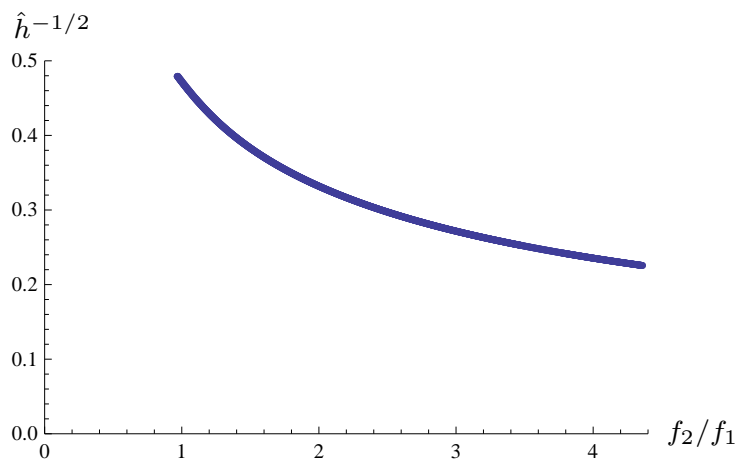

(a) Plot of $\hat{h}^{-1 / 2}$ measuring the scale $\frac{k^{1 / 2}}{2 \pi l_{s}}$.

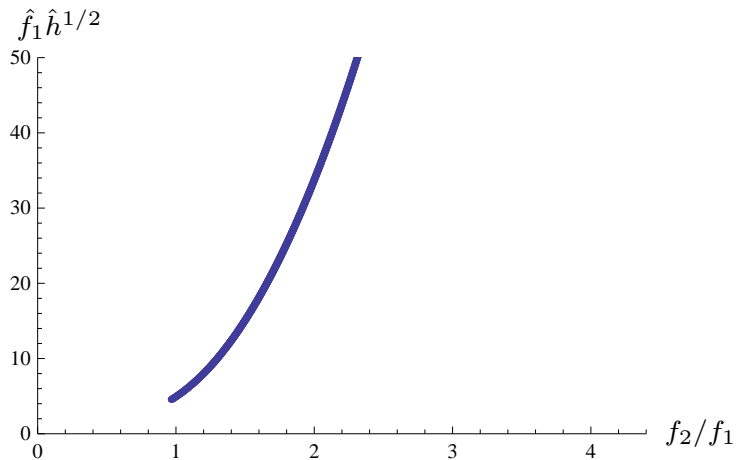

(b) Plot of $\hat{f}_{1} \hat{h}^{1 / 2}$ measuring the dilaton $e^{\Phi}$.

Figure 2: Plots of radius and dilaton for the family of properly quantized dS solutions on $\mathrm{SU}(2) \times \mathrm{SU}(2)$.

We first explained in detail the 4D as well as the 10D approach that have both been used in the literature. Then we classified homogeneous group spaces that are consistent with an SU(3)-structure, extending the previous analyses [9, 55, 56]. We discussed orbifold and orientifold projections of these group spaces that lead to an $\mathcal{N}=1$ supergravity in 4D. Here we paid special attention to the abelian orbifold group $\mathbb{Z}_{2} \times \mathbb{Z}_{2}$ which is (presumably) the only abelian orbifold that can lead to dS extrema. For the first time in the type IIA flux compactification literature we discussed non-abelian orbifolds. We were able to exclude several orbifold groups but also showed that $\Delta(12)$ allows for dS extrema. We classified all group spaces that are compatible with the $\mathbb{Z}_{2} \times \mathbb{Z}_{2}$ or $\Delta(12)$ orbifold groups and that lead to dS extrema. Unfortunately, all the numerical dS extrema we found have at least one tachyonic direction with $\eta \lesssim-2$ so that these solutions are not stable and incompatible with slow-roll inflation. This numerical analysis revealed that semi-simple groups almost always lead to dS extrema (except for $\mathfrak{s o}(3) \times \mathfrak{s o}(2,1)$ as it is not consistent with the symmetries). Furthermore we found many examples amongst solvable algebras of order 3 and one example for a solvable algebra of order 2. Finally there were some examples for algebras that are a semi-direct product of a 3-dimensional solvable algebra with a 3dimensional simple algebra. We could not find dS extrema for any of the nilmanifolds. This is somewhat surprising since nilmanifolds are everywhere negatively curved and negative curvature is a necessary condition for a dS solution. In contrast, $\mathrm{SU}(2) \times \mathrm{SU}(2)$ is only negatively curved in "a corner of its moduli space", but that corner harbors dS extrema.

Finally, we discussed the flux and charge quantization. This has been neglected so far in the literature since the internal spaces have generically a rather complicated geometry which aggravates the analysis. We worked out the flux quantization explicitly for the example of $\mathrm{SU}(2) \times \mathrm{SU}(2) / \mathbb{Z}_{2} \times \mathbb{Z}_{2}$ [8], whose $10 \mathrm{D}$ origin is best understood [19], and found that the small range of parameters that lead to dS extrema are not compatible with flux and charge quantization in the supergravity regime. This presents a great new challenge for all the constructions in the literature. 
Throughout the paper we pointed out many important questions:

- Is it possible to find stable dS vacua or is the tachyonic direction universal:27

- Does the scalar potential allow for slow-roll?

- Is flux and charge quantization generically incompatible with the existence of dS extrema or is this only the case for our explicit $\mathrm{SU}(2) \times \mathrm{SU}(2) / \mathbb{Z}_{2} \times \mathbb{Z}_{2}$ example?

- Can one exclude all non-abelian orbifold groups except $\Delta(12)$ or are other non-abelian orbifold groups compatible with dS extrema?

- Is it possible to include the back-reaction of the O6-planes and how does this affect the solutions [33 35]?

- Is it possible to study compactifications on more general geometries like for example the smooth, compact toric varieties discussed in [76]?

We hope that this paper provides the technical tools necessary to embark on answering some of these questions.

\section{Acknowledgements}

We like to thank David Andriot, Andres Collinucci, Raphael Flauger, Daniel Junghans, Liam McAllister, Susanne Reffert, Daniel Robbins, Mike Stillman, Henry Tye and Marco Zagermann for useful discussions. U.D. is supported by the Swedish Research Council (VR) and U.D. and T.V.R. are supported by the Göran Gustafsson Foundation. P.K. is a Postdoctoral Fellow of the FWO - Vlaanderen. The work of P.K. is further supported in part by the FWO - Vlaanderen project G.0651.11 and in part by the Federal Office for Scientific, Technical and Cultural Affairs through the 'Interuniversity Attraction Poles Programme Belgian Science Policy' P6/11-P. The work of S.S.H. and G.S. was supported in part by NSF CAREER Award No. PHY-0348093, DOE grant DE-FG-02-95ER40896, a Research Innovation Award and a Cottrell Scholar Award from Research Corporation, a Vilas Associate Award from the University of Wisconsin, and a John Simon Guggenheim Memorial Foundation Fellowship. T.W. is supported by the Alfred P. Sloan Foundation and by the NSF under grant PHY-0757868. T.V.R. thanks the University of Wisconsin, Madison for hospitality. G.S. and T.W. thank the Hong Kong Institute for Advanced Study for hospitality.

\footnotetext{
${ }^{27}$ There does not seem to be a simple structure in the tachyonic modes that would allow us to understand why all solutions sofar are unstable. Though some progress has been made to understand the presence of tachyonic modes for de Sitter solutions in four-dimensional supergravity theories 73 75, it is not clear yet how the models with a known higher-dimensional origin, like ours, fit into that discussion.
} 


\section{A Form conventions for reduction to 4D}

Consider type IIA string theory on an SU(3)-structure manifold $M_{6}$, equipped with a $\mathbb{Z}_{2}$ orientifold action which includes an anti-holomorphic involution $\sigma$. The forms on $M_{6}$ then split into even and odd parts, depending upon the behavior of each class under $\sigma$. We will take the following basis of representative real form 28 :

- The zero-form 1 ,

- a set of odd two-forms $Y_{i}^{(2-)}, i=1, \ldots, h_{-}^{1,1}$,

- a set of even two-forms $Y_{\alpha}^{(2+)}, \alpha=1, \ldots, h_{+}^{1,1}$,

- a set of even four-forms $Y^{(4+) i}, i=1, \ldots, h_{-}^{1,1}$,

- a set of odd four-forms $Y^{(4-) \alpha}, \alpha=1, \ldots, h_{+}^{1,1}$,

- a six form $Y^{(6-)}$, odd under $\sigma$,

- a set of even three-forms $Y_{K}^{(3+)}, K=1, \ldots, h^{2,1}+1$,

- and a set of odd three-forms $Y^{(3-) K}, K=1, \ldots, h^{2,1}+1$.

It turns out that we can always choose the $Y_{K}^{(3+)}$ and $Y^{(3-) K}$ to form a symplectic basis such that the only non-vanishing intersections are

$$
\int Y_{K}^{(3+)} \wedge Y^{(3-) J}=\delta_{K}^{J}
$$

Furthermore, we define the triple intersecting numbers

$$
\kappa_{i j k}=\int Y_{i}^{(2-)} \wedge Y_{j}^{(2-)} \wedge Y_{k}^{(2-)}, \quad \hat{\kappa}_{i \alpha \beta}=\int Y_{i}^{(2-)} \wedge Y_{\alpha}^{(2+)} \wedge Y_{\beta}^{(2+)},
$$

and take the even degree forms to satisfy

$$
\int Y^{(6-)}=1, \quad \int Y_{i}^{(2-)} \wedge Y^{(4+) j}=\delta_{i}^{j}, \quad \int Y_{\alpha}^{(2+)} \wedge Y^{(4-) \beta}=\delta_{\alpha}^{\beta}
$$

\section{B Half-flat manifolds}

A six-dimensional SU(3)-structure manifold can be characterized by a globally defined real two-form $J$ and a complex decomposable three-form $\Omega=\Omega_{R}+i \Omega_{I}$, satisfying a compatibility and a normalization condition

$$
\Omega \wedge J=0, \quad \Omega \wedge \Omega^{*}=(4 i / 3) J \wedge J \wedge J=8 i \operatorname{vol}_{6} .
$$

\footnotetext{
${ }^{28}$ The existence of everywhere non-vanishing one-forms would imply that the structure group is a strict subgroup of $\mathrm{SU}(3)$ like for example $\mathrm{SU}(2)$ or the trivial group. We do not consider such cases here.
} 
From the real part of the three-form we can build an almost complex structure for which $J$ is of type $(1,1)$ and $\Omega$ is of type $(3,0)$. It is given by

$$
I_{k}^{l}=c \varepsilon^{m_{1} m_{2} \ldots m_{5} l}\left(\Omega_{R}\right)_{k m_{1} m_{2}}\left(\Omega_{R}\right)_{m_{3} m_{4} m_{5}},
$$

where $\varepsilon$ is the Levi-Civita symbol, and the real scalar $c$ is such that $I$ is properly normalized: $\frac{1}{6} \operatorname{tr}\left(I^{2}\right)=-1$. The metric then follows via

$$
g_{m n}=-I_{m}^{l} J_{l n}
$$

The torsion classes $W_{1}, \ldots, W_{5}$ correspond to the expansion of the exterior derivatives of $J$ and $\Omega$ in terms of SU(3)-representations [77. An SU(3)-structure (that is not also an $\mathrm{SU}(2)$-structure) has no nowhere-vanishing one-forms. If we restrict to left-invariant torsion classes on an homogeneous manifold, this implies that the torsion classes $W_{4}$ and $W_{5}$ have to vanish. In the absence of D-terms the $\mathrm{SU}(3)$-structure manifold is a so-called half-flat manifold [40]. In our conventions this corresponds to $W_{1}, W_{2}$ real. We will often restrict to this case although it would be interesting to study the more general setup. For a half-flat manifold we have

$$
\begin{aligned}
& \mathrm{d} J=\frac{3}{2} W_{1} \Omega_{R}+W_{3}, \\
& \mathrm{~d} \Omega_{R}=0, \\
& \mathrm{~d} \Omega_{I}=W_{1} J \wedge J+W_{2} \wedge J,
\end{aligned}
$$

where $W_{1}$ is a real scalar, $W_{2}$ a real primitive $(1,1)$-form and $W_{3}$ a real primitive $(1,2)+$ $(2,1)$-form. This means that

$$
\begin{array}{ll}
W_{2} \wedge J \wedge J=0, & W_{3} \wedge J=0 \\
W_{2} \wedge \Omega=0, & W_{3} \wedge \Omega=0 .
\end{array}
$$

Furthermore, we find that under the Hodge star, defined from the metric (B.3),

$$
\star_{6} \Omega=-i \Omega, \quad \star_{6} J=\frac{1}{2} J \wedge J, \quad \star_{6} W_{2}=-J \wedge W_{2} .
$$

The Ricci tensor can be expressed in terms of the torsion classes [54, 178]. For that we use that any real symmetric two-tensor $T_{i j}$ splits as follows in representations of $\mathrm{SU}(3)$

$$
T_{i j}=\frac{s\left(T_{i j}\right)}{6} g_{i j}+T_{i j}^{+}+T_{i j}^{-} .
$$

$s\left(T_{i j}\right)$ is the trace, an $\mathrm{SU}(3)$-invariant, and $T_{i j}^{+}$and $T_{i j}^{-}$transform respectively as 8 and $\mathbf{6}+\overline{\mathbf{6}}$. The latter are traceless and have respectively index structure $(1,1)$ and $(2,0)+(0,2)$

$$
\begin{array}{ll}
T_{i j}^{+} g^{i j}=0, & I_{k}^{i} T_{i j}^{+} I_{l}^{j}=T_{k l}^{+}, \\
T_{i j}^{-} g^{i j}=0, & I_{k}^{i} T_{i j}^{-} I^{j}{ }_{l}=-T_{k l}^{-} .
\end{array}
$$


We can associate a primitive real $(1,1)$-form and a complex primitive $(2,1)$-form to respectively $T_{i j}^{+}$and $T_{i j}^{-}$

$$
\begin{aligned}
& P_{2}\left(T_{i j}\right)=\frac{1}{2} J_{i}^{k} T_{k j}^{+} \mathrm{d} x^{i} \wedge \mathrm{d} x^{j}, \\
& P_{3}\left(T_{i j}\right)=\frac{1}{2} T_{i l}^{-} \Omega^{l}{ }_{j k} \mathrm{~d} x^{i} \wedge \mathrm{d} x^{j} \wedge \mathrm{d} x^{k} .
\end{aligned}
$$

Using this it is shown in [54] that the Ricci tensor can be expressed as follows

$$
\begin{aligned}
s\left(R_{i j}\right) & =\frac{15}{2}\left(W_{1}\right)^{2}-\frac{1}{2}\left(W_{2}\right)^{2}-\frac{1}{2}\left(W_{3}\right)^{2}, \\
P_{2}\left(R_{i j}\right) & =-\frac{1}{4} \star\left(W_{2} \wedge W_{2}\right)-\frac{1}{2} \star_{6} d \star_{6}\left(W_{3}-\frac{1}{2} W_{1} \Omega_{R}\right), \\
P_{3}\left(R_{i j}\right) & =\left.2 W_{1} W_{3}\right|_{(2,1)}+\left.2 \mathrm{~d} W_{2}\right|_{(2,1)}-\frac{1}{4} Q_{1}\left(W_{3}, W_{3}\right),
\end{aligned}
$$

with

$$
Q_{1}\left(W_{3}, W_{3}\right)=\left(\Omega^{i j k} \iota_{j} \iota_{i} W_{3} \wedge \iota_{k} W_{3}\right)_{(2,1)},
$$

and where in the right-hand side of eqs. $(\mathrm{B} .10 \mathrm{~b})-(\mathrm{B} .10 \mathrm{c})$ the projection onto the primitive part is understood.

It is also useful to define the following quantity

$$
Q_{2}\left(\hat{W}_{3}, \hat{W}_{3}\right)=\left.\left(\frac{1}{2} \hat{W}_{3 i m n} \hat{W}_{3}^{p m n} \Omega_{p j k} \mathrm{~d} x^{i} \wedge \mathrm{d} x^{j} \wedge \mathrm{d} x^{k}\right)\right|_{(2,1)} .
$$

\section{10D equations of motion for the universal ansatz}

If we plug the universal ansatz (2.14) into the type IIA SUGRA equations of motion and assume the constraint equations (2.16) we find, after a lengthy calculation, the following algebraic equations

$$
\begin{aligned}
& \left.\left(\frac{3}{2} f_{2} W_{1}-\frac{1}{4} f_{3} w_{2}+f_{1} f_{7}+j_{1}\right) \Omega_{R}+\left(f_{2} w_{3}+f_{3} d_{1}+f_{1} f_{8}+j_{2}\right) \hat{W}_{3}=0 \quad \text { (Bianchi } \hat{F}_{2}\right), \\
& \left(3 f_{4} W_{1}+\frac{1}{4} f_{5} w_{2}-f_{6} f_{7}\right) \Omega_{R}+\left(2 f_{4} w_{3}-f_{5} d_{1}-f_{6} f_{8}\right) \hat{W}_{3}=0 \quad\left(\text { eom } \hat{F}_{4}\right), \\
& \left(e^{-2 \Phi} f_{7} W_{1}+e^{-2 \Phi} \frac{f_{8} w_{3}}{6}-\frac{1}{2} f_{1} f_{2}-2 f_{2} f_{4}-\frac{1}{6} f_{3} f_{5}-f_{4} f_{6}\right) J \wedge J \\
& \left.+\left(e^{-2 \Phi} f_{7} w_{2}-e^{-2 \Phi} f_{8} d_{1}+f_{1} f_{3}-2 f_{3} f_{4}+f_{2} f_{5}+f_{3} f_{5} d_{2}-f_{5} f_{6}\right) J \wedge \hat{W}_{2}=0 \quad \text { (C. } 1 \mathrm{~b}\right) \\
& \left.R_{4}=-\frac{15}{2}\left(W_{1}\right)^{2}+\frac{1}{2}\left[\left(w_{2}\right)^{2}+\left(w_{3}\right)^{2}+\left(f_{8}\right)^{2}\right]+2 f_{7}^{2}+2 e^{\Phi} j_{1} \quad \text { (dilaton eom), } H\right)
\end{aligned}
$$




$$
\begin{gathered}
\begin{array}{c}
R_{4}+e^{2 \Phi}\left[\left(f_{1}\right)^{2}+3\left(f_{2}\right)^{2}+\left(f_{3}\right)^{2}+12\left(f_{4}\right)^{2}+\left(f_{5}\right)^{2}+\left(f_{6}\right)^{2}\right]+4 e^{\Phi} j_{1}=0 \\
\quad \text { (external part Einstein eq. }),
\end{array} \\
-2\left(f_{7}\right)^{2}-\frac{1}{2}\left(f_{8}\right)^{2}+\frac{e^{2 \Phi}}{4}\left[5\left(f_{1}\right)^{2}+9\left(f_{2}\right)^{2}+12\left(f_{4}\right)^{2}-\left(f_{6}\right)^{2}+3\left(f_{3}\right)^{2}+\left(f_{5}\right)^{2}\right]+3 e^{\Phi} j_{1}=0 \\
\quad \text { (trace Einstein/dilaton eom), } \\
\begin{array}{c}
\frac{d_{2}\left(w_{2}\right)^{2}}{4}-\frac{d_{1} w_{3}}{2}-\frac{W_{1} w_{2}}{4}+\frac{\left(f_{8}\right)^{2} d_{4}}{2}+\frac{e^{2 \Phi}}{4}\left[\left(\left(f_{5}\right)^{2}-\left(f_{3}\right)^{2}\right) d_{2}+4 f_{2} f_{3}+8 f_{4} f_{5}\right]=0 \\
(\text { Einstein eq. }, \text { two-form part }),
\end{array} \\
\left.2\left(W_{1} w_{3}+d_{1} w_{2}-e^{\Phi} j_{2}-2 f_{7} f_{8}\right) \hat{W}_{3}\right|_{(2,1)}-\frac{1}{4}\left(\left(w_{3}\right)^{2} Q_{1}\left(\hat{W}_{3}, \hat{W}_{3}\right)+\left(f_{8}\right)^{2} Q_{2}\left(\hat{W}_{3}, \hat{W}_{3}\right)\right)=0
\end{gathered}
$$

(Einstein eq., three-form part).

\section{Flux/charge quantization with non-trivial $H$-field}

\section{D.1 General discussion}

In this appendix we study in some detail the quantization of the NSNS flux $H$, the RRfluxes and the charge of the orientifold planes. The easiest is the quantization condition for $H$, which just reads

$$
\frac{1}{\left(2 \pi l_{s}\right)^{2}} \int_{\Sigma^{i}} H=n_{H}^{i}
$$

with $n_{H}^{i}$ integer for all cycles $\Sigma^{i}$ that are non-trivial in homology. Here, $l_{s}=\alpha^{1 / 2}$ is the string length. For the charge of the orientifold plane we have to take into account that the source $j$ entering the Bianchi identities, is in fact given by

$$
j=\sum_{\mathrm{O} p}\left(2 \kappa_{10}^{2}\right) T_{p} m_{p} j_{\mathrm{PD}},
$$

where $2 \kappa_{10}^{2}=(2 \pi)^{7} l_{s}^{8}, T_{p}=(2 \pi)^{-p} l_{s}^{-p-1}$ is the D-brane tension, $m_{p}=-2^{p-5}$ the proportionality between the O-plane and the D-brane tension, and $j_{\mathrm{PD}}$ is the actual Poincaré dual to the submanifold (see [32,79,80] for exact definition and conventions for $j$ ) that the orientifold wraps. For each O-plane wrapping a non-trivial cycle $\Sigma^{i}$, we find, plugging in all the factors, for the O-plane charge

$$
\left(2 \pi l_{s}\right)^{p-7} \int_{\Sigma^{i}} j=m_{p}=-2^{p-5} .
$$

The more subtle part is the quantization of the RR-fluxes, for which, in the presence of non-trivial $H$-flux, one should in fact use $H$-twisted K-theory [81, 82]. However, we will use here the result of [36], where it is claimed that for the case of a simply-connected six manifold $H$-twisted $\mathrm{K}$-theory is isomorphic to $H$-twisted homology. We will study the quantization for the $\mathrm{dS}$ solution on $\mathrm{SU}(2) \times \mathrm{SU}(2)$, which is indeed simply-connected, and use the approach of twisted homology. 
In order to define the twisted boundary operator, let us first consider what constitutes a consistent D-brane [83, 84]. Consider the D-brane's Chern-Simons action,

$$
S_{\mathrm{CS}}=T_{p} \int_{\Sigma} C \wedge e^{\mathcal{F}}
$$

with $\mathcal{F}$ the D-brane world-volume flux, satisfying

$$
\mathrm{d} \mathcal{F}=\left.H\right|_{\Sigma}
$$

This action is only invariant under the gauge transformation of the RR-fields, $\delta C=\mathrm{d}_{H} \Lambda$, if $\Sigma$ has no boundary. So a consistent D-brane should wrap a submanifold without boundary, a cycle. On the other hand, if the D-brane wraps a cycle which is itself a boundary (of some $\Gamma$ ) then its charge is trivial because it can shrink to zero by sweeping out $\Gamma$.

Now, if we introduce non-trivial $H$-field this story is changed in two ways [84]. First of all, as will become clear in a moment, we are forced to consider networks of several D-branes wrapping submanifolds $\Sigma_{k}$ of different dimensions $k$ (but still of the same parity). Secondly, we need to relax eq. (D.5) to allow for magnetic monopoles of the D-brane world-volume field

$$
\mathrm{d} \mathcal{F}=\left.H\right|_{\Sigma_{k}}+\left(2 \pi l_{s}\right)^{2} \delta^{3}\left(\mathcal{C}_{k-3}\right)
$$

where $\mathcal{C}_{k-3}$ is codimension three within $\Sigma_{k}$. In this way we allow the cohomology class of $\left.H\right|_{\Sigma_{k}}$ to be non-trivial. More precisely, the submanifold $\mathcal{C}_{k-3}$ is homologous to the Poincaré dual of $\left.H\right|_{\Sigma_{k}}$ within $\Sigma_{k}$. Revisiting the argument of gauge invariance of the Chern-Simons action eq. (D.4 $)$ we find that $\mathcal{C}_{k-3}$ should be considered in the same way as the boundary of $\Sigma_{k}$. Namely, for a consistent network of D-branes the sum of the ordinary boundaries and these Poincaré duals should vanish. Since the ordinary boundary is of codimension one and the Poincaré dual of codimension three, the necessity of considering networks of D-branes of different dimensions becomes clear. Likewise, there is a new way for a D-brane to decay and thus have trivial charge. Indeed, if it wraps a submanifold corresponding to the magnetic monopole of $\mathcal{F}$ it can dissolve in the larger D-brane. So we are naturally led to consider the homology of the twisted boundary operator. In [36] this operator is defined as follows

$$
\partial_{H}=\partial+H \cap
$$

where $\partial$ is the ordinary boundary operator and $H \cap$ produces the codimension-three Poincaré dual of $\left.H\right|_{\Sigma_{k}}$ within $\Sigma_{k} 29$

So let us now discuss quantization of the RR-fluxes using twisted homology [84]. For each consistent network of D-branes, we want the contribution of the Chern-Simons action to the path integral to be well-defined. Let us write the Chern-Simons term in a manifestly gauge-invariant way as follows. For an even/odd network $\sum_{k \in \mathrm{E} / \mathrm{O}}\left(\Sigma_{k}, \mathcal{F}_{\Sigma_{k}}\right)$ we choose a

\footnotetext{
${ }^{29}$ More accurately, $\mathcal{C}_{k-3}$ is the Poincaré dual of $\left.H\right|_{\Sigma}-\mathrm{d} \mathcal{F}$. In 36 the world-volume gauge field $\mathcal{F}$ is put to zero. This, however, does not affect the homology of the twisted boundary operator as it only depends on the cohomology class of $H$. So we will also put $\mathcal{F}=0$ in the following.
} 
fixed reference network $\sum_{k \in \mathrm{E} / \mathrm{O}}\left(\Sigma_{k}^{0}, \mathcal{F}_{\Sigma_{k}^{0}}\right)$ in the same twisted homology class, such that there exists an odd/even network $\sum_{k \in \mathrm{O} / \mathrm{E}}\left(\tilde{\Gamma}_{k}, \mathcal{F}_{\tilde{\Gamma}_{k}}\right)$ satisfying

$$
\partial_{H}\left(\sum_{k \in \mathrm{O} / \mathrm{E}}\left(\tilde{\Gamma}_{k}, \mathcal{F}_{\tilde{\Gamma}_{k}}\right)\right)=\sum_{k \in \mathrm{E} / \mathrm{O}}\left(\Sigma_{k}, \mathcal{F}_{\Sigma_{k}}\right)-\sum_{k \in \mathrm{E} / \mathrm{O}}\left(\Sigma_{k}^{0}, \mathcal{F}_{\Sigma_{k}^{0}}\right) .
$$

We find then:

$$
S_{\mathrm{CS}}=S_{\mathrm{CS}}^{0}+\sum_{\tilde{\Gamma}_{k}} T_{k-1} \int_{\tilde{\Gamma}_{k}} F \wedge e^{\mathcal{F}_{\tilde{\Gamma}_{k}}}
$$

where the contribution $S_{\mathrm{CS}}^{0}$ from the reference chain is just a constant. We want the contribution of the Chern-Simons action to the path integral,

$$
\exp \left(2 \pi i S_{\mathrm{CS}}\right)
$$

to be independent of the choice of network $\sum_{k \in \mathrm{O} / \mathrm{E}}\left(\tilde{\Gamma}_{k}, \mathcal{F}_{\tilde{\Gamma}_{k}}\right)$. Two such networks would differ by an odd/even $H$-twisted cycle $\sum_{k \in \mathrm{O} / \mathrm{E}}\left(\Gamma_{k}, \mathcal{F}_{\Gamma_{k}}\right)$. So in the end we find the quantization condition

$$
\sum_{k} \frac{1}{\left(2 \pi l_{s}\right)^{k-1}} \int_{\Gamma_{k}} F \wedge e^{\mathcal{F}_{\Gamma_{k}}} \in \mathbb{Z}
$$

for every $H$-twisted cycle $\sum_{k \in \mathrm{O} / \mathrm{E}}\left(\Gamma_{k}, \mathcal{F}_{\Gamma_{k}}\right)$. In words, we need the integral of the RR-fluxes $F$ to be an integer upon integrating over a non-trivial cycle in $H$-twisted homology.

\section{D.2 The $H$-twisted homology of $\mathrm{SU}(2) \times \mathrm{SU}(2)$}

As an example, let us calculate the $H$-twisted homology of $\mathrm{SU}(2) \times \mathrm{SU}(2)$. Let us take

$$
H=\alpha\left(e^{123}-e^{456}\right)
$$

with $\alpha$ some proportionality constant. The non-trivial three-cycles are the two $S^{3} \mathrm{~s} \Sigma_{1}$ and $\Sigma_{2}$. Imposing the quantization of $H$, eq. (D.1), for these two cycles, we find

$$
\frac{1}{\left(2 \pi l_{s}\right)^{2}} \int_{\Sigma_{1}} H=-\frac{1}{\left(2 \pi l_{s}\right)^{2}} \int_{\Sigma_{2}} H=n_{H}
$$

which fixes $\alpha=\frac{n_{H}\left(2 \pi l_{s}\right)^{2}}{C_{S^{3}}}$ with $C_{S^{3}}=\int_{\Sigma_{1}} e^{123}=16 \pi^{2}$.

One can show that $H$-twisted homology is dual to the cohomology of the $H$-twisted exterior derivative [84]

$$
\mathrm{d}_{H}=\mathrm{d}+H \wedge
$$

We can therefore try to calculate the $H$-twisted cohomology first. We know that for a coset manifold, the ordinary cohomology is isomorphic to the cohomology of left-invariant forms (see e.g. [57]). Let us assume that this also applies to $H$-twisted cohomology. One can 
construct a proof of this using the spectral sequence method outlined below. Calculating the $\mathrm{d}_{H^{-}}$-cohomology of left-invariant forms one finds in a straightforward way:

$$
H_{\mathrm{E}}^{H}(\mathrm{SU}(2) \times \mathrm{SU}(2), \mathbb{R})=H_{\mathrm{O}}^{H}(\mathrm{SU}(2) \times \mathrm{SU}(2), \mathbb{R})=\mathbf{1},
$$

where $E / O$ indicate the even respectively odd cohomology 30 However, this is a bit quick, because we need the integer cohomology instead of the real one.

So let us instead calculated the $H$-twisted homology directly. We use the spectral sequence method explained in more detail in [36]. There the twisted homology is calculated by a series of approximations $E_{\mathrm{E}}^{i}$ and $E_{\mathrm{O}}^{i}$ that eventually converges to the correct answer. At each step one defines a differential operator:

$$
\mathrm{d}_{i}: E_{\mathrm{E} / \mathrm{O}}^{i} \longrightarrow E_{\mathrm{O} / \mathrm{E}}^{i}
$$

and the next step in the series is the cohomology of this operator:

$$
E_{\mathrm{E} / \mathrm{O}}^{i+1}=\frac{\operatorname{Ker}\left(\mathrm{d}_{i}: E_{\mathrm{E} / \mathrm{O}}^{i} \rightarrow E_{\mathrm{O} / \mathrm{E}}^{i}\right)}{\operatorname{Im}\left(\mathrm{d}_{i}: E_{\mathrm{O} / \mathrm{E}}^{i} \rightarrow E_{\mathrm{E} / \mathrm{O}}^{i}\right)} .
$$

Now, we take $E_{\mathrm{E} / \mathrm{O}}^{0}$ the sets of even and odd chains and

$$
\mathrm{d}_{1}=\partial, \quad \mathrm{d}_{2}=H \cap .
$$

So $E^{1}$ is in fact the untwisted homology, while for a six-dimensional simply-connected orientable manifold one can show that $E^{2}$ has already converged to the correct answer.

Let us now apply this to $\mathrm{SU}(2) \times \mathrm{SU}(2)$. The untwisted homology is given by

$$
\begin{aligned}
& H_{\mathrm{E}}(\mathrm{SU}(2) \times \mathrm{SU}(2), \mathbb{Z})=\operatorname{span}_{\mathbb{Z}}\left([\{p\}],\left[\Sigma_{1}\right] \times\left[\Sigma_{2}\right]\right) \\
& H_{\mathrm{O}}(\mathrm{SU}(2) \times \mathrm{SU}(2), \mathbb{Z})=\operatorname{span}_{\mathbb{Z}}\left(\left[\Sigma_{1}\right],\left[\Sigma_{2}\right]\right) .
\end{aligned}
$$

Let us now consider the action of $\mathrm{d}_{2}=H \cap$ :

$$
\begin{aligned}
& H \cap[\{p\}]=0, \\
& H \cap\left[\Sigma_{1}\right]=-H \cap\left[\Sigma_{2}\right]=n_{H}[\{p\}] \\
& H \cap\left(\left[\Sigma_{1}\right] \times\left[\Sigma_{2}\right]\right)=\left(H \cap\left[\Sigma_{1}\right]\right) \times\left[\Sigma_{2}\right]-\left[\Sigma_{1}\right] \times\left(H \cap\left[\Sigma_{2}\right]\right)=n_{H}\left(\left[\Sigma_{1}\right]+\left[\Sigma_{2}\right]\right) .
\end{aligned}
$$

So the closed cycles are spanned by $[\{p\}]$ and $\left[\Sigma_{1}\right]+\left[\Sigma_{2}\right]$. On the other we find that the exact cycles are spanned by $n_{H}[\{p\}]$ and $n_{H}\left(\left[\Sigma_{1}\right]+\left[\Sigma_{2}\right]\right)$. It follows that the even and odd $H$-twisted homology is given by

$$
H_{\mathrm{E}}^{H}(\mathrm{SU}(2) \times \mathrm{SU}(2), \mathbb{Z})=\mathbb{Z}_{n_{H}}, \quad H_{\mathrm{O}}^{H}(\mathrm{SU}(2) \times \mathrm{SU}(2), \mathbb{Z})=\mathbb{Z}_{n_{H}}
$$

\footnotetext{
${ }^{30}$ Just as for the D-branes we have to take sums of forms of different dimensions, but of the same parity. So instead of finding a cohomology for every dimension of forms, we just have an even and an odd cohomology.
} 
So we find that there is discrete torsion, which of course we could not see by calculating the real cohomology. As an example, in contrast to ordinary homology in real $H$-twisted homology a point is now not a non-trivial cycle anymore, but is a generalized boundary. In the dual language the volume-form is trivial because it is exact. So it would seem we do not need to quantize the Romans mass $\hat{F}_{0}$ anymore, but

$$
\begin{aligned}
n_{\hat{F}_{0}}=\left(2 \pi l_{s}\right) \hat{F}_{0} & =\left(2 \pi l_{s}\right) \frac{1}{\left(C_{S^{3}}\right)^{2}} \int_{M} \hat{F}_{0} e^{123456} \\
& =\frac{1}{n_{H}} \frac{1}{2 \pi l_{s}} \int_{M} \hat{F}_{0} H \wedge \frac{1}{2 C_{S^{3}}}\left(e^{123}+e^{456}\right) \\
& =-\frac{1}{n_{H}} \frac{1}{2 \pi l_{s}} \int_{\Sigma_{1}} j=\frac{1}{n_{H}} \frac{1}{2 \pi l_{s}} \int_{\Sigma_{2}} j \\
& =-\frac{1}{n_{H}} n_{j}
\end{aligned}
$$

where we used the Bianchi identity $\mathrm{d} \hat{F}_{2}+\hat{F}_{0} H=-j$. So the quantization of $j$ would imply the quantization of $\left(2 \pi l_{s}\right) \hat{F}_{0}$ in units of $1 / n_{H}$. But in fact the integer homology is not trivial but $\mathbb{Z}_{n_{H}}$, which indeed implies the quantization of $\left(2 \pi l_{s}\right) \hat{F}_{0}$ as an integer.

\section{References}

[1] S. Kachru, R. Kallosh, A. D. Linde and S. P. Trivedi, De Sitter vacua in string theory, Phys. Rev. D68 (2003) 046005 hep-th/0301240.

[2] V. Balasubramanian and P. Berglund, Stringy corrections to Kähler potentials, SUSY breaking, and the cosmological constant problem, JHEP 11 (2004) 085 hep-th/0408054.

[3] V. Balasubramanian, P. Berglund, J. P. Conlon and F. Quevedo, Systematics of moduli stabilisation in Calabi-Yau flux compactifications, JHEP 03 (2005) 007 hep-th/0502058.

[4] M. P. Hertzberg, S. Kachru, W. Taylor and M. Tegmark, Inflationary constraints on type IIA string theory, JHEP 12 (2007) 095 [0711.2512].

[5] E. Silverstein, Simple de Sitter Solutions, Phys. Rev. D77 (2008) 106006 [0712.1196].

[6] C. Caviezel, P. Koerber, S. Körs, D. Lüst, D. Tsimpis and M. Zagermann, The effective theory of type IIA AdS $S_{4}$ compactifications on nilmanifolds and cosets, Class. Quant. Grav. 26 (2009) 025014 [0806.3458].

[7] S. S. Haque, G. Shiu, B. Underwood and T. Van Riet, Minimal simple de Sitter solutions, Phys. Rev. D79 (2009) 086005 [0810.5328]. 
[8] C. Caviezel, P. Koerber, S. Körs, D. Lüst, T. Wrase and M. Zagermann, On the cosmology of type IIA compactifications on SU(3)-structure Manifolds, JHEP 04 (2009) 010 [0812.3551].

[9] R. Flauger, S. Paban, D. Robbins and T. Wrase, Searching for slow-roll moduli inflation in massive type IIA supergravity with metric fluxes, Phys. Rev. D79 (2009) 086011 [0812.3886].

[10] U. H. Danielsson, S. S. Haque, G. Shiu and T. Van Riet, Towards classical de Sitter solutions in string theory, JHEP 09 (2009) 114 [0907.2041].

[11] C. Caviezel, T. Wrase and M. Zagermann, Moduli stabilization and cosmology of type IIB on SU(2)-structure orientifolds, JHEP 04 (2010) 011 [0912.3287].

[12] T. Wrase and M. Zagermann, On classical de Sitter vacua in string theory, Fortschr. Phys. 58 (2010) 906-910 [1003.0029].

[13] A. Saltman and E. Silverstein, A new handle on de Sitter compactifications, JHEP 01 (2006) 139 hep-th/0411271.

[14] B. de Carlos, A. Guarino and J. M. Moreno, Flux moduli stabilisation, supergravity algebras and no-go theorems, JHEP 01 (2010) 012 [0907.5580].

[15] B. de Carlos, A. Guarino and J. M. Moreno, Complete classification of Minkowski vacua in generalised flux models, JHEP 02 (2010) 076 [0911.2876].

[16] X. Dong, B. Horn, E. Silverstein and G. Torroba, Micromanaging de Sitter holography, Class. Quant. Grav. 27 (2010) 245020 [1005.5403].

[17] D. Andriot, E. Goi, R. Minasian and M. Petrini, Supersymmetry breaking branes on solvmanifolds and de Sitter vacua in string theory, 1003.3774.

[18] G. Dibitetto, R. Linares and D. Roest, Flux compactifications, gauge algebras and de Sitter, Phys. Lett. B688 (2010) 96-100 [1001.3982].

[19] U. H. Danielsson, P. Koerber and T. Van Riet, Universal de Sitter solutions at tree-level, JHEP 05 (2010) 090 [1003.3590].

[20] G. W. Gibbons and C. M. Hull, de Sitter space from warped supergravity solutions, hep-th/0111072.

[21] R. Kallosh and A. D. Linde, M-theory, cosmological constant and anthropic principle, Phys. Rev. D67 (2003) 023510 hep-th/0208157.

[22] R. Kallosh, A. D. Linde, S. Prokushkin and M. Shmakova, Gauged supergravities, de Sitter space and cosmology, Phys. Rev. D65 (2002) 105016 hep-th/0110089. 
[23] D. Roest and J. Rosseel, De Sitter in extended supergravity, Phys. Lett. B685 (2010) 201-207 [0912.4440].

[24] I. P. Neupane, Compactification on curved manifolds, 1006.4495.

[25] M. J. Duff, B. E. W. Nilsson and C. N. Pope, Kaluza-Klein supergravity, Phys. Rept. 130 (1986) 1-142.

[26] D. Kapetanakis and G. Zoupanos, Coset space dimensional reduction of gauge theories, Phys. Rept. 219 (1992) 1-76.

[27] D. Lüst, Compactification of ten-dimensional superstring theories over Ricci-flat coset spaces, Nucl. Phys. B276 (1986) 220.

[28] L. Castellani and D. Lüst, Superstring compactification on homogeneous coset spaces with torsion, Nucl. Phys. B296 (1988) 143.

[29] T. R. Govindarajan, A. S. Joshipura, S. D. Rindani and U. Sarkar, Supersymmetric compactification of the heterotic string on coset spaces, Phys. Rev. Lett. 57 (1986) 2489 .

[30] D. Cassani and A.-K. Kashani-Poor, Exploiting $N=2$ in consistent coset reductions of type IIA, Nucl. Phys. B817 (2009) 25-57 [0901.4251].

[31] B. S. Acharya, F. Benini and R. Valandro, Fixing moduli in exact type IIA flux vacua, JHEP 02 (2007) 018 hep-th/0607223.

[32] P. Koerber and D. Tsimpis, Supersymmetric sources, integrability and generalized-structure compactifications, JHEP 08 (2007) 082 [0706.1244].

[33] J. Blåbäck, U. H. Danielsson, D. Junghans, T. Van Riet, T. Wrase and M. Zagermann, Smeared versus localised sources in flux compactifications, JHEP 12 (2010) 043 [1009.1877].

[34] J. Blåbäck, U. H. Danielsson, D. Junghans, T. Van Riet, T. Wrase and M. Zagermann, to appear,.

[35] M. R. Douglas and R. Kallosh, Compactification on negatively curved manifolds, JHEP 06 (2010) 004 [1001.4008].

[36] A. Collinucci and J. Evslin, Twisted homology, JHEP 03 (2007) 058 hep-th/0611218.

[37] O. DeWolfe, A. Giryavets, S. Kachru and W. Taylor, Type IIA moduli stabilization, JHEP 07 (2005) 066 hep-th/0505160.

[38] A.-K. Kashani-Poor and R. Minasian, Towards reduction of type II theories on SU(3) structure manifolds, JHEP 03 (2007) 109 hep-th/0611106. 
[39] A.-K. Kashani-Poor, Nearly Kähler reduction, JHEP 11 (2007) 026 [0709.4482].

[40] M. Ihl, D. Robbins and T. Wrase, Toroidal orientifolds in IIA with general NSNS fluxes, JHEP 08 (2007) 043 [0705.3410].

[41] D. Robbins and T. Wrase, D-Terms from generalized NSNS fluxes in type II, JHEP 12 (2007) 058 [0709.2186].

[42] J.-P. Derendinger, C. Kounnas, P. M. Petropoulos and F. Zwirner, Superpotentials in IIA compactifications with general fluxes, Nucl. Phys. B715 (2005) 211-233 hep-th/0411276.

[43] T. W. Grimm and J. Louis, The effective action of type IIA Calabi-Yau orientifolds, Nucl. Phys. B718 (2005) 153-202 [hep-th/0412277].

[44] G. Villadoro and F. Zwirner, $N=1$ effective potential from dual type-IIA D6/O6 orientifolds with general fluxes, JHEP 06 (2005) 047 hep-th/0503169].

[45] T. House and E. Palti, Effective action of (massive) IIA on manifolds with SU(3) structure, Phys. Rev. D72 (2005) 026004 hep-th/0505177.

[46] P. G. Cámara, A. Font and L. E. Ibáñez, Fluxes, moduli fixing and MSSM-like vacua in a simple IIA orientifold, JHEP 09 (2005) 013 hep-th/0506066.

[47] I. Benmachiche and T. W. Grimm, Generalized $N=1$ orientifold compactifications and the Hitchin functionals, Nucl. Phys. B748 (2006) 200-252 hep-th/0602241.

[48] M. Ihl and T. Wrase, Towards a realistic type IIA $T^{6} / \mathbb{Z}_{4}$ orientifold model with background fluxes, JHEP 07 (2006) 027 hep-th/0604087.

[49] G. Villadoro and F. Zwirner, On general flux backgrounds with localized sources, JHEP 11 (2007) 082 [0710.2551].

[50] A. Micu, E. Palti and G. Tasinato, Towards Minkowski vacua in type II string compactifications, JHEP 03 (2007) 104 hep-th/0701173.

[51] M. P. Hertzberg, M. Tegmark, S. Kachru, J. Shelton and O. Ozcan, Searching for inflation in simple string theory models: an astrophysical perspective, Phys. Rev. D76 (2007) 103521 [0709.0002].

[52] D. Lüst and D. Tsimpis, Supersymmetric AdS 4 compactifications of IIA supergravity, JHEP 02 (2005) 027 hep-th/0412250.

[53] P. Koerber and S. Körs, A landscape of non-supersymmetric AdS vacua on coset manifolds, Phys. Rev. D81 (2010) 105006 [1001.0003].

[54] L. Bedulli and L. Vezzoni, The Ricci tensor of SU(3)-manifolds, J. Geom. Phys. 4 (2007) 1125 [math/0606786]. 
[55] P. Koerber, D. Lüst and D. Tsimpis, Type IIA AdS $S_{4}$ compactifications on cosets, interpolations and domain walls, JHEP 07 (2008) 017 [0804.0614].

[56] M. Graña, R. Minasian, M. Petrini and A. Tomasiello, A scan for new $N=1$ vacua on twisted tori, JHEP 05 (2007) 031 hep-th/0609124.

[57] F. Müller-Hoissen and R. Stückl, Coset spaces and ten-dimensional unified theories, Class. Quant. Grav. 5 (1988) 27.

[58] P. Turkowski, Low-dimensional real Lie algebras, J. Math. Phys. 29 (1988) 2139-2144.

[59] P. Turkowski, Solvable Lie algebras of dimension six, J. Math. Phys. 31 (1990) 1344-1350.

[60] D. Roest, M-theory and gauged supergravities, Fortsch. Phys. 53 (2005) 119-230 hep-th/0408175.

[61] M. de Roo, D. Westra and S. Panda, De Sitter solutions in $N=4$ matter coupled supergravity, JHEP 0302 (2003) 003 hep-th/0212216].

[62] M. de Roo, D. Westra, S. Panda and M. Trigiante, Potential and mass matrix in gauged $N=4$ supergravity, JHEP 0311 (2003) 022 hep-th/0310187.

[63] G. Dall'Agata, G. Villadoro and F. Zwirner, Type-IIA flux compactifications and $N=4$ gauged supergravities, JHEP 08 (2009) 018 [0906.0370].

[64] G. Dibitetto, A. Guarino and D. Roest, Charting the landscape of $N=4$ flux compactifications, 1102.0239 .

[65] P. Fré, M. Trigiante and A. Van Proeyen, Stable de Sitter vacua from $N=2$ supergravity, Class. Quant. Grav. 19 (2002) 4167-4194 hep-th/0205119.

[66] H. F. Blichfeldt, Finite collineation groups. The Univ. Chicago Press, Chicago, 1917.

[67] A. Hanany and Y.-H. He, Non-abelian finite gauge theories, JHEP 02 (1999) 013 hep-th/9811183.

[68] P. O. Ludl, Comments on the classification of the finite subgroups of SU(3), 1101.2308 .

[69] S. Reffert, Toroidal orbifolds: resolutions, orientifolds and applications in string phenomenology, hep-th/0609040.

[70] S. Reffert, The geometer's toolkit to string compactifications, 0706.1310.

[71] J. Gray, Y.-H. He, A. Ilderton and A. Lukas, STRINGVACUA: a Mathematica package for studying vacuum configurations in string phenomenology, Comput. Phys. Commun. 180 (2009) 107-119 [arXiv:0801.1508]. 
[72] J. M. Maldacena and C. Núñez, Supergravity description of field theories on curved manifolds and a no go theorem, Int. J. Mod. Phys. A16 (2001) 822-855 hep-th/0007018.

[73] L. Covi et al., de Sitter vacua in no-scale supergravities and Calabi-Yau string models, JHEP 06 (2008) 057 [0804.1073].

[74] M. Gómez-Reino, J. Louis and C. A. Scrucca, No metastable de Sitter vacua in $N=2$ supergravity with only hypermultiplets, JHEP 0902 (2009) 003 [arXiv:0812.0884].

[75] A. Borghese and D. Roest, Metastable supersymmetry breaking in extended supergravity, 1012.3736.

[76] M. Larfors, D. Lüst and D. Tsimpis, Flux compactification on smooth, compact three-dimensional toric varieties, JHEP 07 (2010) 073 [1005.2194].

[77] S. Chiossi and S. Salamon, The intrinsic torsion of $S U(3)$ and $G_{2}$ structures, Ann. Mat. Pura e Appl. 282 (1980) 35-58 math/0202282.

[78] T. Ali and G. B. Cleaver, The Ricci curvature of half-flat manifolds, JHEP 05 (2007) 009 hep-th/0612171.

[79] P. Koerber and L. Martucci, Deformations of calibrated D-branes in flux generalized complex manifolds, JHEP 12 (2006) 062 hep-th/0610044.

[80] P. Koerber, Lectures on Generalized Complex Geometry for physicists, Fortsch. Phys. 59 (2011) 169-242 [1006.1536].

[81] G. W. Moore and E. Witten, Self-duality, Ramond-Ramond fields, and K-theory, JHEP 05 (2000) 032 hep-th/9912279.

[82] R. Minasian and G. W. Moore, K-theory and Ramond-Ramond charge, JHEP 11 (1997) 002 hep-th/9710230.

[83] J. Evslin, What does(n't) K-theory classify?, hep-th/0610328.

[84] J. Evslin and L. Martucci, D-brane networks in flux vacua, generalized cycles and calibrations, JHEP 07 (2007) 040 hep-th/0703129. 